\title{
BREXIT E DIRITTO INTERNAZIONALE PRIVATO TRA ROMA, BRUXELLES E LUGANO: "HOW CAN I JUST LET YOU WALK AWAY ?"
}

\author{
BREXIT AND PRIVATE INTERNATIONAL LAW, BETWEEN \\ ROME, BRUSSELS AND LUGANO: "HOW CAN I JUST LET \\ YOU WALK AWAY?"
}

\author{
BERNARDO CORTESE \\ Professore Ordinario di Diritto dell'Unione europea \\ Università di Padova. Dipartimento di Diritto Pubblico, Internazionale e Comunitario / \\ Scuola di Giurisprudenza
}

Recibido: 23.09.2020 / Aceptado: 05.11.2020

DOI: https://doi.org/10.20318/cdt.2021.5956

\begin{abstract}
Riassunto: Quali sfide pone Brexit per il diritto internazionale privato in materia civile e commerciale, nei rapporti tra UE e Regno Unito? Come affrontare quelle sfide? L'articolo cerca di dare risposta a queste domande, con riferimento ai Regolamenti Roma I, Roma II, Bruxelles I(bis) e alla Convenzione di Lugano del 2007. In tale contesto sono analizzate le regole e i principi giuridici applicabili tanto nella fase del periodo transitorio, quanto in quella successiva. In particolare, si considerano le prospettive di un cliff-edge scenario al termine del periodo transitorio, individuando nell'ambito della giurisdizione $\mathrm{i}$ profili in cui il traffico giuridico rischia di subire una pesante compromissione. Il lavoro si chiude con alcune proposte per un percorso negoziale capace di depotenziare quel rischio.
\end{abstract}

Parole chiave: diritto internazionale privato, giurisdizione, riconoscimento delle sentenze, Brexit.

Abstract: What challenges does Brexit pose for private international law in civil and commercial matters, in EU/UK cases? How to address those challenges? The article attempts to answer these questions, with reference to the Rome I, Rome II, Brussels I(bis) Regulations and the 2007 Lugano Convention. In this context, the rules and legal principles applicable both during the transition period and afterwards are analysed. In particular, the prospects of a cliff-edge scenario at the end of the transition period are considered; jurisdiction and recognition of decisions are identified as areas in which legal traffic risks being heavily compromised. The article puts forward some proposals for a negotiating path capable of depotentiating that risk.

Keywords: private international law, jurisdiction, recognition of judgments; Brexit

Sommario: I. Introduzione. 1. Premessa... semiseria. 2. Piano del lavoro. II. Il recesso. 3. L'Accordo di recesso dall'Unione: la perdurante applicazione del diritto dell'Unione nel periodo transitorio. 4. Segue: Il periodo transitorio e gli accordi internazionali conclusi dall'Unione. 5. Le conseguenze del recesso sul diritto dello Spazio Economico Europeo (e sulla Convenzione di Lugano). III. Il diritto internazionale privato dell'Unione e la sua applicazione al Regno Unito durante il periodo transitorio. 6. I regolamenti Roma I e Roma II. 7. La Convenzione di Roma del 1980 sulla legge applicabile alle obbligazioni contrattuali. 8. Il regolamento Bruxelles I (bis). 9. ...e le convenzioni su giurisdizione e riconoscimento: la Convenzione di Bruxelles del 1968... 10. ...la Convenzione di Lugano del 2007. 11. Le convenzioni dell'Aja del 2005 e del 2019. IV. Cliff-edge scenario. 
12. Una biforcazione. 13. Il diritto applicabile: verso una continuità delle regole. 14. Giurisdizione e riconoscimento delle sentenze: l'impossibile reviviscenza degli accordi bilaterali e l'urgenza di un accordo su una convention double. 15. Il rischio di conflitti di giurisdizione: a) i principi. 16. Segue: b) Il concreto modificarsi dei titoli di giurisdizione. 17. Gli ostacoli al riconoscimento delle sentenze britanniche nell'Unione. 18. ...e gli ostacoli al riconoscimento delle sentenze continentali nel Regno Unito. 19. Ritorno sulla necessità di instaurare un quadro di collaborazione attorno ad una convention double. 20. L'insufficienza degli strumenti dell'Aja. 21. L'adozione di un regime unilaterale britannico modulato sul regime di Bruxelles / Lugano come possibile soluzione ponte. 22. ...completata da un parallelo regime speciale UE. V. Conclusioni. 23. Conclusioni operative: Il percorso per un possibile accordo ad hoc. 24. Conclusioni semiserie: As you like it...

\section{Introduzione}

1. "How can I just let you walk away / Just let you leave without a trace? / (...) And you coming back to me is against all odds"1. Scherzosamente, si potrebbe iniziare con queste strofe di una famosa canzone di Phil Collins quasi ogni pezzo su Brexit: di certo, uno su Brexit e diritto internazionale privato da una prospettiva continentale.

In fondo, Brexit, come la canzone di Collins, è la storia di un fallimento... amoroso. Del resto, il paradigma del divorzio è presto entrato nel linguaggio istituzionale di Brexit, almeno in riferimento alla definizione degli obblighi finanziari del Regno Unito in relazione agli impegni assunti, pro quota, in quanto membro dell'Unione ${ }^{2}$. Si tratta qui di un'unione (intesa come rapporto) lunga, per quanto riguarda il Regno Unito, mezzo secolo. Uno dei partner di quell'unione si era certo stancato quasi subito del rapporto, ma rimaneva, pur ostile e riottoso, nella casa comune: il suo effettivo allontanarsi, infine, produce incredulità, insieme ad una residua, tenue speranza che il legame possa rinsaldarsi, contro ogni aspettativa.

Come spesso accade nei divorzi, del resto, anche in questo caso tutto rischia finire danneggiato dal rancore di fondo: anche quegli aspetti del vivere insieme che sembravano non porre grandi problemi. Ed è questo il caso, in particolare, della partecipazione del Regno Unito al diritto internazionale privato dell'Unione: una partecipazione che, a parte qualche scossone (forum non conveniens, anti-suit injunctions...) sembrava procedere in modo più che tollerabile, nell'interesse di tutti ${ }^{3}$.

2. Dopo questa premessa semiseria, proviamo a mettere le carte in tavola, inquadrando i temi giuridici oggetto del presente lavoro. L'intenzione è di occuparci qui dell'influsso del recesso del Regno Unito dall'Unione sul diritto internazionale privato dell'Unione, in materia civile e commerciale: per l'essenziale, i regimi relativi alla legge applicabile alle obbligazioni contrattuali ed extracontrattuali, da una parte, e quelli in materia di giurisdizione e riconoscimento delle decisioni, dall'altra. Per poter abbozzare un'analisi in materia, però, sarà anzitutto necessario fare i conti con il recesso nel suo insieme e, per l'essenziale, con l'Accordo di recesso tra l'Unione e il Regno Unito che ancora ci dà solo una serie di prospettive, ma nessuna certezza, se non di brevissimo termine. A ciò è dedicata, per l'essenziale, la prima sezione del presente lavoro. Il passaggio successivo ci porterà ad esaminare, nella seconda sezione, il curioso status quo determinato, in questo come in quasi tutti gli ambiti del diritto dell'Unione, dall'estensione di un periodo transitorio durante il quale uno Stato terzo, il Regno Unito, è considerato come se fosse ancora membro. Nella terza sezione, ci interrogheremo sugli scenari per il periodo in cui l'uscita del Regno Unito dall'orbita del diritto dell'Unione si sarà definitivamente prodotta. In particolare, proveremo a ragionare sui modi in cui i rischi di un cliff-edge scenario potranno essere mitigati, specie nell'area in cui quello scenario appare destinato a produrre gli effetti più significativi: quella della giurisdizione e del riconoscimento delle decisioni giudiziarie.

\footnotetext{
${ }^{1}$ Phillip David Charles Collins, Against All Odds (1984).

${ }^{2}$ Cfr. European Commission, "Position paper transmitted to the UK: essential principles on the financial settlement", 12 giugno 2017, accessibile on-line $<$ https://ec.europa.eu/commission/publications/position-paper-essential-principles-financial-settlement_en> (visto in luglio 2020). Il c.d. Brexit Bill è l'oggetto della parte V dell'Accordo di recesso (infra, nota 4).

${ }^{3}$ Cfr. però le pesanti critiche di T.C. HaRtLey, "The European Union and the Systematic Dismantling of the Common Law Conflict of Laws", International and Comparative Law Quarterly, Vol. 54, 2005, pp. 813-828.
} 


\section{Il recesso}

3. L'accordo sui termini del recesso del Regno Unito dall'Unione europea, tanto faticosamente entrato in vigore alla mezzanotte del 31 gennaio $2020^{4}$, risolve solo temporaneamente le questioni legate all'uscita del Regno Unito dall'Unione.

Esso infatti prevede un periodo transitorio durante il quale il Regno Unito, pur essendo uno Stato terzo ai sensi del diritto UE, continuerà ad applicare il diritto UE e ad essere equiparato ad uno Stato membro, ai fini dell'applicazione del vigente diritto $\mathrm{UE}^{5}$ - fatto salvo l'intervenuto esaurimento di tutti i diritti e poteri di cui il Regno Unito godeva nell'ambito del sistema istituzionale dell'Unione in quanto Stato membro ${ }^{6}$.

Il periodo transitorio è destinato a scadere il 31 dicembre $2020^{7}$, salva una proroga frutto di accordo tra le due parti, che prenda la forma di decisione del "comitato misto" responsabile dell'attuazione dell'Accordo nei rapporti tra le parti contraenti ${ }^{8}$, da adottarsi entro il 1. luglio $2020^{9}$.

Poiché quella proroga non è stata convenuta nei termini previsti dall'Accordo, in linea di principio si deve assumere che il 31 dicembre 2020 sia ormai la data definitiva per il raggiungimento di un accordo che eviti la c.d. hard Brexit.

È però vero che nulla, in questo contesto negoziale degno di un dramma Shakespeariano, può escludersi con definitiva certezza... e così varrà la pena di mantenere aperta una porticina sull'eventualità che un prolungamento del periodo transitorio avvenga comunque. In fin dei conti, l'elasticità che caratterizza il diritto internazionale - oramai l'unico quadro in cui, da un punto di vista giuridico formale, si svolgono le relazioni tra il Regno Unito e l'Unione europea - consentirebbe di raggiungere un accordo novatore, sul punto, dell'Accordo di recesso, purché esso sia, per quanto riguarda il profilo della sua liceità interna nel diritto dell'Unione, concluso con le stesse modalità previste dall'art. 50, par. 2, TUE.

4. Un'ulteriore notazione s'impone circa gli accordi internazionali dell'Unione, che tanto rilievo hanno anche in relazione al diritto internazionale privato.

A tal riguardo, l'Accordo di recesso prevede anzitutto, al suo art. 2, lettera a), che si debbano intendere "diritto dell'Unione", ai fini di quell'Accordo, anche "gli accordi internazionali di cui l'Unione è parte e gli accordi internazionali conclusi dagli Stati membri a nome dell'Unione" (punto iv), nonché "gli accordi tra gli Stati membri conclusi in quanto Stati membri dell'Unione" (punto v).

Ciò posto, l'Accordo di recesso stabilisce, all'art. 129, par. 1, che "durante il periodo di transizione il Regno Unito è vincolato dagli obblighi derivanti dagli accordi internazionali conclusi dall'Unione, dagli Stati membri a nome dell'Unione o dall'Unione e dagli Stati membri congiuntamente".

Ora, se non pone in sé problemi l'assunzione, in forza di un trattato internazionale qual è 1'Accordo di recesso, di un obbligo a favore di terzi (da cui sorga, eventualmente, un diritto per questi) ${ }^{10}$, più dubbia è l'efficacia della precisazione, contenuta alla nota a piè di pagina 137 del testo ufficiale

\footnotetext{
${ }^{4}$ Agreement on the withdrawal of the United Kingdom of Great Britain and Northern Ireland from the European Union and the European Atomic Energy Community, GUUE, n. C 384I, del 12 novembre 2019. Il titolo italiano dell'accordo è "Accordo sul recesso del Regno Unito di Gran Bretagna e Irlanda del Nord dall'Unione europea e dalla Comunità europea dell'energia atomica". L'Accordo sarà indicato in questo lavoro come l' 'Accordo di recesso». L'Accordo, finalmente approvato dalla House of Commons il 9 gennaio 2020 nel testo chiuso a livello di negoziatori il 17 ottobre 2019, dopo gli insuccessi della precedente versione davanti al Parlamento britannico, è stato firmato il 24 gennaio dall'Unione e dal governo britannico, per esser poi formalmente ratificato dal Regno Unito il 29 gennaio, approvato dal Parlamento europeo in quello stesso giorno, ed infine concluso per l'Unione dal Consiglio il 30 gennaio 2020. L'Accordo è entrato in vigore alla mezzanotte del 31 gennaio 2020.

${ }^{5}$ Accordo di recesso, Art. 127, parr. 3 e 6, salve le (relativamente numerose) deroghe specificamente previste nello stesso accordo.

${ }^{6}$ Accordo di recesso, secondo considerando ed art. 7 (oltre a varie altre disposizioni, che ne riprendono il senso in ambiti specifici e che non si ritiene opportuno qui richiamare).

${ }^{7}$ Accordo di recesso, art. 126.

${ }^{8}$ Accordo di recesso, art. 164 .

${ }^{9}$ Accordo di recesso, art. 132, par. 1.

${ }^{10} \mathrm{Cfr}$. a tale proposito, l'art. 36 della Convenzione di Vienna sul diritto dei trattati, ove si afferma che il consenso dello Stato terzo - considerato in quella sede necessario al sorgere di un diritto previsto a suo favore da un trattato concluso da altri - si presume.
} 
dell'Accordo, secondo cui l'Unione comunica "alle altre parti dei suddetti accordi che durante il periodo di transizione, ai fini degli stessi, il Regno Unito va considerato Stato membro".

In fondo, rispetto agli obblighi che Stati terzi hanno assunto nei confronti dell'Unione e del Regno Unito in quanto Stato membro di quella, si pretende con tale dichiarazione unilaterale - ché tale essa è nei confronti delle altre parti - di indurre un temporaneo effetto di successione a favore del Regno Unito, che di quegli accordi non è parte contraente a titolo individuale. Ora, ci si trova in una situazione che presenta manifeste analogie, dal punto di vista del diritto internazionale generale, con quella del distacco di uno Stato dal soggetto (normalmente uno Stato, qui l'Unione) che abbia originariamente concluso un trattato con un terzo Stato ${ }^{11}$. Sul punto sembra allora prudente muovere dall'assunto che tale successione, anche se temporanea, potrà aversi unicamente sul presupposto di una non opposizione delle altre parti contraenti ${ }^{12}$. Ciò, oltre a desumersi per analogia dallo stato della (pur molto variegata) prassi internazionale generale relativa alla secessione/dissoluzione ${ }^{13}$, trova conferma nella specifica prassi che si sta concretamente producendo in relazione agli effetti della Brexit sugli accordi bilaterali con la Svizzera $^{14} \mathrm{e}$ in quella relativa all'Accordo AELS/EFTA, di cui si dirà poco oltre ${ }^{15}$.

Infine, sempre in relazione alle conseguenze della Brexit sulla dimensione esterna dell'azione dell'Unione nel periodo transitorio, è rilevante nel presente contesto notare che l'Accordo di recesso consente al Regno Unito svolgere le attività diplomatiche necessarie ad organizzare le sue future relazioni pattizie con Stati terzi - nonostante la sua assimilazione ad uno Stato membro per il resto dei profili rilevanti. All'art. 129, par. 4, di quell'Accordo si prevede infatti che il Regno Unito possa "negoziare, firmare e ratificare gli accordi internazionali cui ha aderito a proprio nome negli ambiti di competenza esclusiva dell'Unione, purché tali accordi non entrino in vigore né si applichino durante il periodo di transizione, salvo autorizzazione dell'Unione".

5. Con il recesso dall'Unione, il Regno Unito ha altresì cessato di far parte dell'Accordo sullo Spazio Economico Europeo (SEE). Si tratta di un Accordo c.d. misto, concluso cioè dall'allora Comunità e dai suoi Stati membri, da una parte, e dagli Stati membri dell'Associazione Europea di Libero Scambio (AELS/EFTA), dall'altra ${ }^{16}$. Poiché quell'accordo è lo strumento per rendere applicabile agli Stati AELS/ EFTA una parte importante delle libertà del mercato interno, vincolandoli al tempo stesso all'acquis, il Regno Unito e gli Stati AELS/EFTA hanno ritenuto opportuno regolare specificamente le conseguenze sull'Accordo SEE del recesso del Regno Unito dall'Unione, ed hanno concluso uno specifico Accordo sulle conseguenze che il recesso del Regno Unito produce sulle relazioni con gli Stati AELS/EFTA ${ }^{17}$,

\footnotetext{
${ }^{11}$ Per tale parallelo, a dire il vero piuttosto naturale alla luce della dimensione sovranazionale e costituzionale del processo di integrazione europea, v. già M. WAIBEL, "Brexit and Acquired Rights", University of Cambridge Faculty of Law Research Paper No. 61/2017, accessibile su SSRN all'indirizzo https://ssrn.com/abstract=3080210, poi anche in American Journal of International Law Unbound, 2018, pp. 440-444, p. 442; G. SACERDOTI, "Il regime degli scambi del Regno Unito con 1'Unione Europea e i Paesi terzi dopo la Brexit: opzioni e vincoli internazionali", Rivista di diritto internazionale, 2018, pp. 685-714, p. 704; S. VeZZANI, "Gli effetti del recesso dell'Unione europea sui trattati conclusi dall'Unione europea e dallo Stato recedente", Rivista di diritto internazionale, 2019, pp. 1040-1070, p. 1048 ss.

${ }^{12}$ Diversamente da quanto sostenuto da chi ritiene applicabile, per analogia, l'art. 34 della Convenzione di Vienna sulla successione degli Stati nei trattati: cfr., con riferimento alla partecipazione del Regno Unito ad accordi dell'ambito WTO conclusi unicamente dall'Unione, L. BARTELS, "The UK's Status in the WTO after Brexit" (September 23, 2016), accessibile su SSRN all'indirizzo http://dx.doi.org/10.2139/ssrn.2841747, p. 19. Nel senso invece della necessità di ottenere il consenso degli Stati terzi co-contraenti si veda M. Dougan, "An Airbag for the Crash Test Dummies? EU-UK Negotiations for a Post-Withdrawal "status quo" Transitional Regime under Article 50 TEU”, Common Market Law Review, Vol. 56, 2018, pp. 57-99, p. 97.

${ }^{13} \mathrm{Su}$ cui in passato la stessa Corte di giustizia si è espressa (ed alla quale ha in effetti contribuito) con la sentenza 18 novembre 2003, causa C-216/01, Budéjovický Budvar, Raccolta 2003, p. I-13617. Su quella sentenza e sui suoi rapporti con la prassi degli Stati F. CASOLARI, "La successione degli Stati nei trattati bilaterali al vaglio della Corte di giustizia: il caso Budvar", Rivista di diritto internazionale, 2005, pp. 121-148: ivi anche riferimenti al dibattito dottrinale.

${ }^{14}$ La perdurante applicazione degli accordi bilaterali UE/Svizzera anche al Regno Unito è stata l'oggetto di uno specifico scambio di note tra Svizzera e UE: cfr. la nota informativa del governo federale elvetico accessibile su https://www.sem.admin. $\mathrm{ch} / \mathrm{sem} / \mathrm{it} /$ home/aktuell/news/2020/2020-01-310.html (visto in luglio 2020).

${ }^{15}$ Infra, par. 5.

${ }^{16}$ Accordo sullo Spazio economico europeo, fatto ad Oporto il 2 maggio 1992, ed entrato in vigore il primo gennaio 1994, ai sensi del suo art. 129, GUCE n. L 1 del 3 gennaio 1994, p. 3.

${ }^{17}$ Agreement on arrangements between Iceland, the Principality of Liechtenstein, the Kingdom of Norway and the United
} 
cosiddetto Separation Agreement, non ritenendo sufficiente il generale meccanismo di continuità - salvo obiezioni sollevate dalle parti terze - previsto per il periodo transitorio nell'Accordo di recesso ${ }^{18}$.

Collegata alla partecipazione all'Accordo sullo Spazio Economico Europeo (e all'Unione, per gli Stati membri di questa) è anche la partecipazione alla Convenzione di Lugano del 2007, che ha sostituito la precedente convenzione di Lugano del 1988. Ad essa non è fatto alcun riferimento espresso né nell'Accordo di recesso, né nel Separation Agreement.

In relazione a tale convenzione, i contatti tra il Regno Unito e le parti contraenti "terze" hanno però confermato l'impostazione di temporanea continuità, auspicata dall'Unione al già menzionato art. 129, par. 1, dell'Accordo di recesso, cosicché il Regno Unito continuerà a beneficiare dell'applicazione della Convenzione di Lugano durante il periodo transitorio ${ }^{19}$.

\section{Il diritto internazionale privato dell'Unione e la sua applicazione al Regno Unito durante il periodo transitorio}

6. Ciò posto da un punto di vista generale, durante il periodo transitorio - che esso duri soltanto fino al 31 dicembre 2020, o che sia rocambolescamente prorogato oltre quella data...against all odds - gli strumenti del diritto internazionale privato e processuale dell'Unione continueranno ad applicarsi tanto davanti ai giudici del Regno Unito, quanto davanti ai giudici degli Stati membri dell'Unione.

In particolare, secondo quanto disposto all'art. 66 dell'Accordo di recesso, il Regolamento Roma I sulla legge applicabile alle obbligazioni contrattuali ${ }^{20}$ continuerà ad applicarsi ai contratti conclusi dopo il 17 dicembre $2009^{21}$, ma "prima della fine del periodo di transizione", mentre il Regolamento Roma II sulla legge applicabile alle obbligazioni extracontrattuali ${ }^{22}$ continuerà ad applicarsi "ai fatti che danno origine a un danno, se verificatisi a partire dall' 11 gennaio $2009^{23}$, ma prima della fine del periodo di transizione".

Diversamente da quanto accadrebbe in caso di applicazione di quegli strumenti in quanto retained legislation ${ }^{24}$ in assenza di accordo, per le fattispecie considerate dal regime transitorio disposto dall'Accordo di recesso il Regno Unito dovrà intendersi come "Stato membro" ai fini dell'applicazione di quei due regolamenti, secondo quanto previsto in linea generale dall'art. 7 dell'Accordo.

Ciò vale anzitutto, per l'essenziale, in relazione alle previsioni dell'art. 7 del Regolamento Roma I, in materia di legge applicabile ai contratti di assicurazione, nella parte in cui quelle previsioni si riferiscono a "rischi situati in uno Stato membro". Ancora, l'equiparazione ad uno Stato membro rileva altresì nel contesto dell'art. 6, par. 3, lett. b) del Regolamento Roma II, in relazione agli atti limitativi della libera concorrenza, laddove si prevede che all'azione di risarcimento danni possa applicarsi il di-

Kingdom of Great Britain and Northern Ireland following the withdrawal of the United Kingdom from the European Union, the EEA Agreement and other agreements applicable between the United Kingdom and the EEA EFTA States by virtue of the United Kingdom's membership of the European Union, fatto a Londra il 28 gennaio 2020, accessibule su https://assets. publishing.service.gov.uk/government/uploads/system/uploads/attachment_data/file/844413/2019-11-04_Updated_UK-EEA EFTA_Separation_Agreement_.docx (visto in luglio 2020). L'accordo sarà qui di seguito indicato come Separation Agreement.

${ }_{18}$ Previsto dall'art. 129, par. 1, su cui v. supra, al par. 4 di questo lavoro.

${ }^{19} \mathrm{Si}$ veda la nota informativa dell'Ufficio federale di giustizia elvetico, accessibile alla pagina https://www.bj.admin.ch/ bj/it/home/wirtschaft/privatrecht/lugue-2007/brexit-auswirkungen.html, e la corrispondente nota stampa 28 gennaio del Ministry of Justice del Regno Unito su https://www.gov.uk/government/news/support-for-the-uks-intent-to-accede-to-the-luganoconvention-2007 (viste in luglio 2020).

${ }^{20}$ Regolamento (CE) n. 593/2008 del Parlamento europeo e del Consiglio del 17 giugno 2008, sulla legge applicabile alle obbligazioni contrattuali (Roma I), in GUUE n. L 177 del 4 luglio 2008, p. 6.

${ }^{21}$ Secondo quanto previsto dall'art. 28 del Regolamento Roma I.

${ }^{22}$ Regolamento (CE) n. 864/2007 del Parlamento europeo e del Consiglio, dell'11 luglio 2007, sulla legge applicabile alle obbligazioni extracontrattuali (Roma II), GUUE n. L 199 del 31 luglio 2007, p. 40.

${ }^{23}$ Secondo quanto previsto dagli artt. 31 e 32 del Regolamento Roma II.

${ }^{24}$ Di cui si dirà meglio infra al par. 13. Sulla scelta del legislatore britannico di incorporare, per l'essenziale, i regimi previsti dai due regolamenti, nel futuro diritto internazionale privato del Regno Unito in quanto "retained legislation" cfr. A BRIGGS, "Brexit and Private International Law: An English Perspective", Rivista di diritto internazionale privato e processuale, 2019, pp. 261-283, p. 279. 
ritto del giudice adito, corrispondente al domicilio del convenuto o di uno dei convenuti, ove la lesione della concorrenza "interessi direttamente e sostanzialmente anche il mercato dello Stato membro" di quel giudice, in relazione al singolo convenuto o a ciascuno dei convenuti.

Altrettanto deve dirsi con riferimento alle ipotesi più generali di limitazione della portata della scelta di legge nei due regolamenti, per le fattispecie in cui "tutti gli (altri) elementi pertinenti alla situazione siano ubicati, nel momento in cui si opera la scelta, in uno o più Stati membri" 25 .

Per il resto, evidentemente, i due Regolamenti non pongono problemi di questo tipo, avendo in linea di principio portata universale ${ }^{26}$.

7. Fermo restando quanto previsto nell'Accordo di recesso in relazione al Regolamento Roma I, vi sono due contesti, rispettivamente temporale e spaziale, in cui deve ritenersi che continui la vigenza della Convenzione di Roma del 1980 sulla legge applicabile alle obbligazioni contrattuali ${ }^{27}$, nonostante il silenzio mantenuto sul punto da quell'Accordo. Prima di esaminarli con un minimo di attenzione, credo però sia importante fare chiarezza sul persistente vigore, da un punto di vista generale, di quello strumento pattizio, e sulla sua estraneità alle previsioni (ad un tempo novative e dissolutive) dell'Accordo di recesso.

Anzitutto, si deve osservare che la Convenzione, entrata in vigore per il primo gruppo di Stati contraenti il $1^{\circ}$ aprile $1991^{28}$, dopo il primo decennio di vigore si rinnova tacitamente di cinque anni in cinque anni, salvo denuncia ${ }^{29}$. Nessuno degli Stati che ne sono divenuti parte ha presentato tale denuncia. Essa è, dunque, uno strumento di diritto internazionale pattizio tuttora in vigore tra alcuni degli Stati membri dell'Unione $^{30} \mathrm{e}$ il Regno Unito. Quest'ultimo, in particolare, non è vincolato da quella convenzione (come successivamente modificata) nei confronti degli Stati che hanno aderito all'Unione a partire dal 2004.

Ciò posto, è dubbio che ad essa possa considerarsi applicabile la previsione dell'art. 2, lettera a), punto v), dell'Accordo di recesso. In quella disposizione si fa riferimento, come si è ricordato, agli "accordi tra gli Stati membri conclusi in quanto Stati membri dell'Unione", dei quali si afferma la natura di diritto dell'Unione, ai fini dell'Accordo di recesso.

Ora, a me sembra che quella previsione abbia un senso unicamente come espressione del tentativo (probabilmente vano) di tenere in vita la cooperazione rafforzata sul brevetto unitario, che si basa,

\footnotetext{
${ }^{25}$ Art. 3, par. 4 del Regolamento Roma I; art. 14, par. 3, del Regolamento Roma II.

${ }^{26}$ Art. 2 del Regolamento Roma I; art. 3 del Regolamento Roma II.

${ }^{27}$ Convenzione sulla legge applicabile alle obbligazioni contrattuali, aperta alla firma a Roma il 19 giugno 1980, GUCE n. L 266 del 9 ottobre 1980, entrata in vigore ai termini del suo art. 29 il primo aprile 1991, come in seguito modificata dalla Convenzione relativa all'adesione della Repubblica ellenica alla convenzione sulla legge applicabile alle obbligazioni contrattuali, firmata a Lussemburgo il 10 aprile 1984, e dalla Convenzione relativa all'adesione del Regno di Spagna e della Repubblica portoghese, firmata a Funchal il 18 maggio 1992, come ulteriormente completata dal Primo Protocollo concernente l'interpretazione da parte della Corte di giustizia della convenzione sulla legge applicabile alle obbligazioni contrattuali e dal Secondo Protocollo che attribuisce alla Corte di giustizia delle Comunità europee alcune competenze per l'interpretazione della convenzione sulla legge applicabile alle obbligazioni contrattuali, entrambi fatti a Bruxelles il 19 dicembre 1988, GUCE n. L 48 del 20 febbraio 1989, ed ulteriormente modificata dalla Convenzione relativa all'adesione della Repubblica d'Austria, della Repubblica di Finlandia e del Regno di Svezia alla convenzione sulla legge applicabile alle obbligazioni contrattuali, aperta alla firma a Roma il 19 giugno 1980, nonché al primo e al secondo protocollo relativi all'interpretazione da parte della Corte di giustizia, fatta a Bruxelles il 29 novembre 1996, GUCE n. C 15 del 15 gennaio 1997.

${ }^{28}$ Comunicazione del Consiglio 91/C 52/01, in GUCE n. C 52 del 28 febbraio 1991, p. 1.

${ }^{29}$ Convenzione di Roma, art. 30.

${ }^{30}$ Va notato che non tutti gli Stati membri dell'Unione sono parti contraenti di quella convenzione e delle varie convenzioni che ne hanno esteso l'ambito di applicazione (ed introdotto alcune modifiche) nei loro rapporti reciproci. La convenzione è in vigore tra Belgio, Francia, Germania, Grecia, Italia, Lussemburgo e Regno Unito a partire dal 1. aprile 1991. È in vigore dalla stessa data anche per la Danimarca, ma solo per ciò che riguarda la convezione iniziale, tra le sue parti contraenti, e la convenzione di adesione della Grecia. È entrata in vigore successivamente per i Paesi Bassi il 1. settembre 1991, per 1'Irlanda il 1. gennaio 1992: anche in tal caso solo per la convezione iniziale, tra le sue parti contraenti, e la convenzione di adesione della Grecia. Nella versione modificata dalla convenzione di adesione di Spagna e Portogallo essa è in vigore per la Spagna e i Paesi Bassi dal 1. settembre 1993, per l'Italia dal 1. marzo 1994, per il Portogallo dal 1. settembre 1994, e a partire da date successive per Belgio, Germania, Grecia, Francia, Lussemburgo e Regno Unito (dal 1. dicembre 1997). La convenzione di adesione di Austria, Finlandia e Svezia è entrata in vigore per quei Paesi il 1. Aprile 1999, ma essa vincola il Regno Unito, nei rapporti con quelli, solo dal 1. gennaio 2001. In date variabili tra il 2006 e il 2008 è entrata poi in vigore la convenzione di adesione del 2005 dei 10 nuovi Stati membri entrati nell'Unione nel 2004, mentre il 15 gennaio 2008 è entrata in vigore la convenzione di adesione di Bulgaria e Romania. Il Regno Unito non è parte contraente né dell'una né dell'altra di queste due ultime convenzioni.
} 
per gli aspetti giurisdizionali, sull'Accordo su un Tribunale unificato dei brevetti ${ }^{31}$, nel quale il Regno Unito è designato come parte necessaria ${ }^{32} \mathrm{e}$ che costituisce, in quanto strumento internazionale di completamento della cooperazione rafforzata, un mezzo necessario al funzionamento di questa (e dunque di una parte di quel "diritto dell'Unione" mantenuto in vigore ai sensi dell'Accordo di recesso).

La Convenzione di Roma, per contro, non ha un legame necessario con il diritto dell'Unione in senso proprio. È, certo, una convenzione "comunitaria", poiché funzionalmente legata alla Convenzione di Bruxelles del 1968 sulla giurisdizione e il riconoscimento delle decisioni in materia civile e commerciale. Diversamente da quella, però, essa non era prevista dall'allora art. $220 \mathrm{CEE}$, né è più funzionale al buon funzionamento (presente e futuro) del regime UE della giurisdizione, essendo stata soppiantata, nell'ambito di applicazione del diritto dell'Unione, dal Regolamento Roma I.

Del resto, considerare applicabile a quella convenzione l'Accordo di recesso, attraverso il suo art. 2, sarebbe ad un tempo inutile e dannoso, oltre che inopportuno.

Sarebbe inutile, perché la sua perdurante applicazione, nelle due limitate dimensioni in cui è prevista, non dipende da una previsione esterna con effetto novativo della sua vigenza oltre la data di effetto della dichiarazione di recesso del Regno Unito, ma discende dalla Convenzione stessa e dalle previsioni del Regolamento, espressione della volontà dei contraenti, pienamente rilevante alla luce del diritto internazionale generale dei trattati ${ }^{33}$. Sarebbe, una siffatta interpretazione dell'art. 2 dell'Accordo di recesso, altresì inopportuna e dannosa, perché implicherebbe un effetto dissolutivo della Convenzione al termine del periodo transitorio, e ciò sulla base di un intento meramente implicito delle parti contraenti, Regno Unito in primis.

Per quest'ultimo, del resto, vi è piuttosto un importante indizio contrario all'ipotesi di una cessazione del vigore di quella convenzione al termine del periodo transitorio in forza del suddetto art. 2 dell'Accordo. La Convenzione di Roma è infatti considerata ancora applicabile nella normativa interna predisposta dal Regno Unito, a livello esecutivo, in vista di un no-dea ${ }^{34}$.

${ }^{31}$ In GUUE n. C 175 del 20 giugno 2013, p. 1. L'accordo è fatto a Bruxelles il 19 febbraio 2013 ed aperto alla firma e all'adesione degli Stati membri dell'Unione (art. 84).

${ }^{32}$ Argomenta ex art. 87, che pone come condizione per l'entrata in vigore dell'accordo la ratifica di tredici Stati membri che comprendano i tre Stati membri "nei quali il maggior numero di brevetti europei aveva effetto nell'anno precedente a quello in cui ha luogo la firma dell'accordo": in linguaggio meno diplomatico... Germania, Regno Unito e Francia.

${ }^{33}$ Il riferimento obbligato è agli artt. 30, par. 3, 31, par. 3, e 39 della convenzione di Vienna sul diritto dei trattati. Ai sensi dell'art. 30, relativo all'applicazione di trattati successivi relativi alla stessa materia, "[w]hen all the parties to the earlier treaty are parties also to the later treaty but the earlier treaty is not terminated or suspended in operation under article 59 , the earlier treaty applies only to the extent that its provisions are compatible with those of the later treaty". Certo, qui non ci confrontiamo con il succedersi nel tempo di due trattati, ma la norma esprime un principio senz'altro applicabile anche nel nostro caso. Ciò è confermato dalla seconda disposizione sopra citata, ai sensi della quale, nell'interpretazione di un trattato, si tiene conto di "(a) Any subsequent agreement between the parties regarding the interpretation of the treaty or the application of its provisions; (...); (c) Any relevant rules of international law applicable in the relations between the parties." È chiaro che l'adozione nel contesto del sistema dei Trattati dell'Unione - con la partecipazione di tutti gli Stati contraenti della Convenzione - del Regolamento Roma I, atto destinato in quel sistema a prevalere su ogni diversa regola altrimenti vigente nel suo ambito di applicazione, e contenente regole specificamente dirette a istituire una "successione" di regimi tra lo stesso Regolamento e la Convenzione (si veda oltre nel testo), esprime sia un "subsequent agreement" relativo all'applicazione della Convenzione, sia un contesto di "relevant rules of international law applicable in the relations between the parties", cosicché le disposizioni della Convenzione relative al suo ambito di applicazione vanno oramai interpretate alla luce delle citate disposizioni del Regolamento. Al medesimo risultato porta la considerazione della regola generale espressa dall'art. 39 della Convenzione di Vienna, in relazione alla modifica dei trattati, la quale è sottoposta all'accordo delle parti: è evidente che nelle procedure che hanno portato all'adozione del Regolamento Roma I si è espresso, anche se con modalità peculiari, un chiaro accordo delle parti contraenti della Convenzione di Roma ad acconsentire ad una sua modifica coerente con quanto disposto sul punto dal Regolamento. Non convince invece la lettura effettuata da P. BERTOLI, "La "Brexit" e il diritto internazionale privato e processuale", Rivista di diritto internazionale privato e processuale, 2017, pp. 599-632, p. 610, con rinvio ai ragionamenti già svolti dall'A. alla p. 608, secondo cui la Brexit rappresenterebbe un cambiamento fondamentale delle circostanze tale da determinare il venir meno dell'applicazione della Convenzione di Roma (come pure della Convenzione di Bruxelles) al Regno Unito. Per quanto riguarda in particolare la Convenzione di Roma, infatti, a me pare che il fatto stesso che la si sia mantenuta in vigore per dei territori ai quali non si applica il diritto dell'Unione, manifesti che la circostanza dell'appartenenza all'Unione non è, per l'appunto, fondamentale alla perdurante vigenza di quel regime convenzionale.

${ }^{34}$ The Law Applicable to Contractual Obligations and Non-Contractual Obligations (Amendment etc.) (EU Exit) Regulations 2019, Section 3. 
Venendo agli ambiti nei quali le regole della Convenzione di Roma devono ritenersi ancora operanti, e cominciando dal profilo (inter)temporale determinato dal succedersi di Convenzione e Regolamento Roma I, va anzitutto ricordato che la Convenzione si applica ai contratti conclusi dopo la sua entrata in vigore per lo Stato davanti ai cui giudici sia invocata ${ }^{35}$, mentre il Regolamento si applica a quelli conclusi dopo il termine iniziale di efficacia in esso previsto ${ }^{36}$. Pertanto, la Convenzione continuerà ad applicarsi, sia davanti ai tribunali degli Stati membri dell'Unione che ne sono parti contraenti, sia davanti a quelli del Regno Unito, per i contratti conclusi tra il $1^{\circ}$ aprile 1991 (o la successiva data di entrata in vigore per gli Stati che vi hanno aderito in tempi più recenti) e il 17 dicembre 2009, termine iniziale di efficacia del Regolamento.

In secondo luogo, dal punto di vista dell'ambito territoriale di applicazione, va notato che il Regolamento Roma I, ai sensi del suo art. 24, ha sì inteso per larga parte sostituire la Convenzione, ma ne ha fatto altresì salva la perdurante applicazione a "i territori degli Stati membri che rientrano nel campo di applicazione territoriale (della) convenzione", cui però il regolamento non è applicabile, a norma dell'art. 299 TCE, ora art. 355 TFUE. Si tratta per l'essenziale di Gibilterra ${ }^{37}$, di alcune dipendenze francesi (Polinesia francese, Nuova Caledonia e relative dipendenze, terre australi ed antartiche francesi) ${ }^{38}$, di Aruba e delle Antille olandesi ${ }^{39}$ e delle Isole Færøer/Faroe ${ }^{40}$. In quei territori non si applicano i Trattati europei, secondo quanto previsto dall'art. 355 TFUE, che ne determina l'associazione all'Unione in virtù di un regime peculiare ${ }^{41}$, cosicché ad essi non si applica il Regolamento Roma I.

Quei territori hanno dunque contribuito a mantenere attuale quella Convenzione, con i relativi protocolli di interpretazione della Corte di giustizia ${ }^{42}$, almeno sul piano del diritto internazionale. Rimane distinto (e potenzialmente problematico) il profilo della concreta applicazione di quelle regole di diritto internazionale privato uniforme di fronte ai giudici di alcuni Stati membri: vale in particolare il riferimento all'ordinamento tedesco, in cui la Convenzione non operava per forza di rinvio, ma per incorporazione negli art. $34 \mathrm{e}$ seguenti EGBGB. Con l'abrogazione di quegli articoli ${ }^{43} \mathrm{e}$ in considerazione dell'approccio rigorosamente "dualista" dell'ordinamento tedesco al momento dell'applicazione interna delle convenzioni internazionali rimane difficile immaginare l'effettiva applicazione da parte dei giudici tedeschi di quel regime convenzionale, nonostante il suo perdurante vigore internazionale.

\footnotetext{
${ }^{35}$ Convenzione di Roma, art. 17.

${ }^{36}$ Regolamento Roma I, art. 28: il termine in esso previsto è il 17 dicembre 2009.

${ }^{37}$ In forza della dichiarazione presentata dal governo del Regno Unito in conformità all'art. 27, par. 2, lett. b) della Con-
} venzione, la Convenzione si applica a Gibilterra a partire dal 18 luglio 1994. La dichiarazione è accessibile tramite il registro elettronico del Segretariato generale del Consiglio, alla pagina https://www.consilium.europa.eu/en/documents-publications/ treaties-agreements/agreement/?id=1988024 (visto in luglio 2020).

${ }^{38}$ Ai quali la convenzione di Roma è applicabile in virtù della dizione impiegata dal suo art. 27 che, se per gli altri Stati fa riferimento al loro territorio europeo, per la Francia ne dichiara l'applicazione "all'insieme del territorio della Repubblica francese",

${ }^{39}$ In forza della dichiarazione presentata dal governo del Regno dei Paesi Bassi in conformità all'art. 27, par. 2, lett. b) della Convenzione, questa si applica a Aruba e alle Antille Olandesi nella versione modificata dalla convenzione di adesione della Grecia a partire dal 1. Agosto 1993; per quanto riguarda la versione modificata dalla convenzione di adesione di Spagna e Portogallo, si applica a quei territori dal 1. dicembre 2010, in forza di una nuova, specifica dichiarazione. Le dichiarazioni sono accessibili tramite il registro elettronico del Segretariato generale del Consiglio, la prima alla pagina già segnalata supra, alla nota 37, la seconda alla pagina https:/www.consilium.europa.eu/en/documents-publications/treaties-agreements/ratification/?i $\mathrm{d}=1992082$ \&partyid $=$ NL (visto in luglio 2020).

${ }^{40}$ In forza della dichiarazione presentata dal governo danese in conformità all'art. 27, par. 2, lett. b) della Convenzione, la Convenzione si applica alle Faroe a partire dal 1. settembre 1986. La dichiarazione è accessibile tramite il registro elettronico del Segretariato generale del Consiglio, alla pagina https://www.consilium.europa.eu/en/documents-publications/treaties-agreements/agreement/?id=1988024 (visto in luglio 2020).

${ }^{41}$ Definito da ultimo nella Decisione 2013/755/UE del Consiglio del 25 novembre 2013, relativa all'associazione dei paesi e territori d'oltremare all'Unione europea, GUUE n. L 344 del 19 dicembre 2013.

${ }^{42} \mathrm{Si}$ tratta del Primo Protocollo concernente l'interpretazione da parte della Corte di giustizia della convenzione sulla legge applicabile alle obbligazioni contrattuali, aperta alla firma a Roma il 19 giugno 1980, e del Secondo Protocollo che attribuisce alla Corte di giustizia delle Comunità europee alcune competenze per l'interpretazione della convenzione sulla legge applicabile alle obbligazioni contrattuali, aperta alla firma a Roma il 19 giugno 1980, in GUCE n. L 48 del 20 febbraio 1989 , rispettivamente a p. 1 e p. 17, entrambi sono in vigore per il Regno Unito a far data dal 1. agosto 2004.

${ }^{43}$ Gesetz zur Anpassung der Vorschriften des Internationalen Privatrechts an die Verordnung (EG) Nr. 593/2008 vom 25.06.2009, Bundesgesetzblatt, 2009, Parte I, p. 1574. 
Tuttavia, alla luce della scelta del Regno Unito di mantenere un regime costruito sul Regolamento Roma I come retained legislation, ed in considerazione altresì della portata universale del regime regolamentare, la perdurante vigenza della Convenzione non pare destinata a trasformarsi in un passaggio chiave per il mantenimento dell'allineamento del diritto internazionale privato britannico a quello $\operatorname{continentale}^{44}$, ma continuerà piuttosto ad avere un valore estremamente ridotto nella pratica.

8. Ai sensi dell'art. 67 dell'Accordo di recesso, la giurisdizione in materia civile e commerciale è definita, sia nel Regno Unito, sia negli Stati membri dell'Unione "in situazioni che coinvolgano il Regno Unito", dalle previsioni del Regolamento $1215 / 2012^{45}$, c.d. Bruxelles I(bis).

Il riferimento alle situazioni che coinvolgano il Regno Unito va inteso in relazione alle seguenti ipotesi: i) convenuti domiciliati nel Regno Unito ${ }^{46}$; ii) consumatori, siano essi attori o convenuti, domiciliati in quello Stato e parti di un contratto rientrante nell'ambito di applicazione della sezione 4 del capo II del Regolamento ${ }^{47}$; iii) controparti professionali di consumatori le quali, pur non risultando domiciliate in uno Stato membro, abbiano comunque "una succursale, un'agenzia o qualsiasi altra sede d'attività" nel Regno Unito ${ }^{48}$; iv) datori di lavoro che, pur non risultando domiciliati in uno Stato membro, abbiano comunque "una succursale, un'agenzia o qualsiasi altra sede d'attività" nel Regno Unito ${ }^{49}$; v) lavoratori che, agendo contro un datore che sia domiciliato in uno Stato membro o si trovi in una situazione assimilata, abbiano il loro domicilio nel Regno Unito, ovvero che in quello Stato svolgano abitualmente la loro attività, o in quello Stato siano stati assunti, non svolgendo la loro attività abitualmente in un solo Paese - in tali casi, anche laddove il datore non sia domiciliato in uno Stato membro né si trovi in una situazione a ciò assimilabile ${ }^{50}$; vi) titoli di giurisdizione esclusiva riferiti al territorio del Regno Unito ${ }^{51}$; vii) clausole di proroga della giurisdizione a favore dei giudici del Regno Unito ${ }^{52}$, o rugelose Einlassung davanti ai giudici del Regno Unito da parte di un convenuto (domiciliato in uno Stato membro) nei cui confronti non potrebbe stabilirsi altrimenti la giurisdizione ${ }^{53}$; viii) obbligo per l'autorità giurisdizionale britannica di sospendere d'ufficio il procedimento in caso di previa litispendenza davanti al giudice di uno Stato membro (e viceversa); ix) misure cautelari richieste ai giudici del Regno Unito e destinati a produrre effetti in quel territorio ${ }^{54}$, nonché (in termini di proibizione) le ingiunzioni richieste a quei giudici contro l'esercizio della giurisdizione da parte di giudici di Stati membri (anti-suit injunctions) ${ }^{55}$.

Quanto alla dimensione di riconoscimento ed esecuzione delle decisioni, l'Accordo di recesso prevede che le relative disposizioni del Regolamento Bruxelles I(bis) si applicheranno, nel Regno Unito o, negli Stati membri "in situazioni che coinvolgano" quello Stato, al riconoscimento ed esecuzione di decisioni (giudiziarie) "emesse nelle azioni proposte prima della fine del periodo di transizione", nonché ad atti pubblici e transazioni giudiziarie rispettivamente redatti o registrati, ed approvate, prima di quel termine.

A tale proposito va anzitutto segnalata la soluzione adottata in relazione alle decisioni giudiziarie: tenuto conto della durata, non sempre breve, dei processi nei diversi Stati membri, tale scelta appare

\footnotetext{
${ }^{44}$ Come invece ipotizzava, prima delle modifiche introdotte dal Withdrawal Act e dall'Accordo di Recesso, A. Dickinson, "Back to the Future: The UK's EU Exit and the Conflict of Laws", Journal of Private International Law, Vol. 12, 2016, pp. 195-210, p. 204.

${ }^{45}$ Regolamento (UE) n. 1215/2012 del Parlamento europeo e del Consiglio, del 12 dicembre 2012, concernente la competenza giurisdizionale, il riconoscimento e l'esecuzione delle decisioni in materia civile e commerciale, in GUUE n. L 351 del 20 dicembre 2012 , p. 1.

${ }^{46} \mathrm{Cfr}$. in particolare gli artt. 4, 5 e 6 del Regolamento Bruxelles I(bis)

${ }^{47}$ Secondo quanto previsto all'art. 17, par. 1 del Regolamento stesso.

${ }^{48}$ Regolamento Bruxelles I(bis), art. 17, par. 2.

${ }^{49}$ Regolamento Bruxelles I(bis), art. 20, par. 2.

${ }^{50}$ Regolamento Bruxelles I(bis), art. 21.

${ }^{51}$ Regolamento Bruxelles I(bis), art. 24.

${ }^{52}$ Regolamento Bruxelles I(bis), art. 25.

${ }^{53}$ Regolamento Bruxelles I(bis), art. 26.

${ }^{54}$ Regolamento Bruxelles I(bis), art. 35: argomenta dalla sentenza della Corte di giustizia 17 novembre 1998, causa C-391/95, Van Uden Maritime, Raccolta. p. I-7091, punti 40, 47-48.

${ }^{55}$ Sentenza della Corte (seduta plenaria) 27 aprile 2004, causa C-159/02, Turner c. Grovit, Raccolta, 2004, p. I-3565; Sentenza della Corte (grande sezione) del 10 febbraio 2009, causa C-185/07, Allianz SpA e Generali Assicurazioni Generali SpA c. West Tankers Inc., Raccolta, 2009, p. I-663.
} 
adeguata, perché capace di garantire la stabilità e prevedibilità delle situazioni giuridiche ben più del principio generale tempus regit actum che, altrimenti, si sarebbe potuto applicare in materia processuale. La soluzione prescelta va del resto incontro alle preoccupazioni di proteggere i diritti acquisiti dai singoli nel contesto della vigenza del sistema dei Trattati comunitari e poi dell'Unione ${ }^{56}$. Che si scelga invece di rimanere aderenti a quel principio generale per gli atti pubblici appare privo di controindicazioni. Rimane piuttosto discutibile l'ancoraggio al principio tempus regit actum delle transazioni giudiziarie, cui forse avrebbe giovato una soluzione omogenea a quella delle decisioni giudiziarie: in questo modo, di fatto, si rischia di disincentivare il ricorso ad uno strumento di soluzione anticipata di controversie che siano iniziate in sede giudiziaria contenziosa, ma in cui le parti potrebbero giungere ad un accordo à l'amiable senza attendere la decisione giudiziaria.

Quanto alla formulazione relativa all'ambito di applicazione della norma intertemporale così adottata, si sarebbe forse potuta con maggiore trasparenza adottare una dizione diretta: gli Stati membri sono tenuti ad applicare le norme del regolamento sul riconoscimento non in generiche "situazioni che coinvolgano" il Regno Unito, bensì in relazione a decisioni giudiziarie "emess[e] da un'autorità giurisdizionale" di quello Stato ${ }^{57}$, compresi i provvedimenti provvisori ma esclusi quelli adottati inaudita altera parte ${ }^{58}$, ad atti pubblici ivi formalmente redatti o registrati come tali ${ }^{59} \mathrm{e}$ alle transazioni giudiziarie che siano state "approvate" da un'autorità giurisdizionale di quello Stato. Rimane il dubbio se, con la formula prescelta, si escludano dalla disciplina intertemporale prevista dal regolamento le transazioni giudiziarie che non siano state approvate da quelle autorità, ma semplicemente "concluse davanti" ad esse: poiché ad entrambe le ipotesi si applica invece il regolamento ${ }^{60}$.

9. L'Accordo di recesso rimane silente sulla convenzione di Bruxelles del 1968 in materia di giurisdizione e riconoscimento delle decisioni in materia civile e commerciale. Non è, nondimeno, difficile intenderne il destino, che si dimostra marginale per quanto attiene alla perdurante dimensione di applicazione intertemporale e inesistente per quanto attiene ai profili di applicazione territoriale.

Dal punto di vista intertemporale, la convenzione, di cui i Regolamenti Bruxelles I e I(bis) sono l'evoluzione in chiave comunitaria, è applicabile, nella parte in cui definisce i titoli di giurisdizione degli Stati contraenti, solo alle domande giudiziali proposte sino al $1^{\circ}$ marzo 2002, data di entrata in vigore del Regolamento 44/2001, c.d. Bruxelles I ${ }^{61}$ : per le domande introdotte successivamente a quella data, si applica oramai il Regolamento 1215/2012, Bruxelles I(bis), che ha abrogato il precedente Regolamento Bruxelles I, sostituendolo in toto ${ }^{62}$.

Quella stessa data spartiacque determina il riconoscimento secondo la Convenzione, o invece secondo il regime regolamentare, degli atti pubblici, in relazione al momento della loro formazione.

Quanto al riconoscimento ed all'esecuzione delle decisioni giudiziarie, il Regolamento Bruxelles I(bis) si applica alle decisioni che si pronunciano su domande proposte posteriormente all'entrata in vigore del Regolamento 44/2001, ma anche al riconoscimento delle decisioni relative a domande giudiziali pur proposte in data anteriore, ove la decisione da riconoscere sia stata emessa posteriormente a quella data, purché la domanda giudiziale sia stata introdotta dopo l'entrata in vigore, sia nello Stato di origine che in quello richiesto, di almeno una delle due convenzioni di Bruxelles del 1968 o di Lugano del 1988, o, in ogni caso, ove la giurisdizione sia stata affermata sulla base di titoli corrispondenti a

\footnotetext{
${ }^{56}$ Se ne veda una discussione in M. WAIBEL, "Brexit and Acquired Rights", cit., p. 442 s. Per la discussione del diverso ma parallelo caso della giurisdizione internazionale in relazione al recesso di uno Stato da un trattato che preveda la giurisdizione di un'istanza internazionale per la soluzione di controversie fra Stati v. M. Buscemi, L. MarotTI, "Obblighi procedurali e conseguenze del recesso dai trattati: quale rilevanza della convenzione di Vienna nella prassi recente?", Rivista di diritto internazionale, 2019, pp. 939-976, p. 962 ss.

${ }^{57} \mathrm{Cfr}$. i termini adottati all'art. 2, lettera a) del Regolamento Bruxelles I(bis) per definire il proprio ambito di applicazione.

${ }^{58}$ Argomenta da Regolamento Bruxelles I(bis), art. 2, lettera a), secondo comma.

${ }^{59}$ Regolamento Bruxelles I(bis), art. 2, lettera c).

${ }^{60}$ Regolamento Bruxelles I(bis), art. 2, lettera b).

${ }^{61}$ Regolamento (CE) n. 44/2001 del Consiglio del 22 dicembre 2000 concernente la competenza giurisdizionale, il riconoscimento e l'esecuzione delle decisioni in materia civile e commerciale, GUCE n. L 12 del 16 gennaio 2001, se ne vedano gli artt. 66, quanto all'applicabilità ratione temporis, e 76, quanto all'entrata in vigore.

${ }^{62}$ Regolamento Bruxelles I(bis), art. 80.
} 
quelli previsti nel regolamento, o sia basata su una convenzione comunque in vigore tra Stato di origine e Stato richiesto ${ }^{63}$.

In ultima analisi, dunque, l'ambito di residua applicazione della Convenzione di Bruxelles nel profilo intertemporale si riduce al lumicino.

In relazione poi al profilo dell'applicabilità del regime convenzionale a territori non coperti dall'art. 355 TFUE e sottoposti alla sovranità di alcuni Stati membri o del Regno Unito - non dissimile nei presupposti da quello esaminato più sopra per la Convenzione di Roma - la disciplina della Convenzione di Bruxelles è stata integralmente sostituita dalla "nuova" convenzione di Lugano, quella cioè conclusa nel 2007 per aggiornare il testo originario del 1988 alle modifiche già introdotte, all'epoca, nel primo strumento di formattazione comunitaria della Convenzione di Bruxelles, il Regolamento 44/2001: dispone chiaramente in tal senso l'art. 69, par. 7 della Convenzione di Lugano ${ }^{64}$.

Né potrebbe invocarsi, contro tale conclusione, il considerando n. 9 del Regolamento Bruxelles I bis, secondo cui la Convenzione del 1968 continuerebbe ad applicarsi a quei territori. Infatti, da un lato, ad un considerando non può attribuirsi valore precettivo, ma unicamente interpretativo; dall'altro lato, opinare diversamente ne implicherebbe l'invalidità, per violazione di una previsione posta da fonte superiore, quale è la Convenzione del 2007, conclusa dall'Unione con gli Stati AELS/EFTA.

Non convince, pertanto, la tesi di chi vedrebbe nel momento della Brexit (senza accordo) una reviviscenza per il Regno Unito della Convenzione del $1968^{65}$, poiché quella tesi si basa sull'ormai superato presupposto del continuato vigore di quel trattato in relazione ai territori extraeuropei di cui s'è detto. Cosicché anche il riferimento contenuto nella Section 2(1) del Civil Jurisdiction and Judgments Act $1982^{66}$, da ritenersi sicuramente espressione di un rinvio formale e non di una recezione materiale, non avrebbe più altro significato che in relazione al profilo intertemporale.

10. Venendo ora alla Convenzione di Lugano, non si trova alcun diretto riferimento ad essa né nell'Accordo di recesso, che si occupa invece dei regolamenti UE in materia di diritto internazionale privato $^{67}$, né nel c.d. Separation Agreement concluso tra il Regno Unito e gli Stati AELS/EFTA.

Essa è dunque genericamente sottoposta all'impostazione di continuità salvo obiezioni che si può desumere dall'art. 129, par. 1, dell'Accordo di recesso ${ }^{68}$.

Di tale convenzione, che sostituisce integralmente la precedente convenzione di Lugano del 1988, il Regno Unito non è parte a titolo individuale. Come è noto, infatti, sulla base del (pur criticabile) ${ }^{69}$

\footnotetext{
${ }^{63}$ Regolamento Bruxelles I, art. 66, par. 2, letto alla luce dell'art. 80 del Regolamento Bruxelles I(bis).

64 “Per quanto riguarda le relazioni tra gli Stati membri della Comunità europea e i territori non europei di cui all'articolo 70, paragrafo 1, lettera b), la presente convenzione sostituisce la convenzione concernente la competenza giurisdizionale e l'esecuzione delle decisioni in materia civile e commerciale, firmata a Bruxelles il 27 settembre 1968, e il protocollo relativo all'interpretazione di detta convenzione da parte della Corte di giustizia delle Comunità europee, firmato a Lussemburgo il 3 giugno 1971, modificati dalle convenzioni di adesione a detta convenzione e a detto protocollo da parte degli Stati aderenti alle Comunità europee, a decorrere dalla data di entrata in vigore della presente convenzione nei confronti di quei territori a norma dell'articolo 73, paragrafo 2".

${ }^{65}$ A. Dickinson, "Back to the future....", cit., p. 204 s.; R. Airens, A. Dinsmore, "Jurisdiction, Enforcement and the Conflict of Laws in Cross-Border Commercial Disputes: What Are the Legal Consequences of Brexit?", European Business Law Review, 2016, pp. 903-920, p. 907.

${ }^{66}$ Valorizzato in R. Airens, A. Dinsmore, op. cit., p. 906 ss.

${ }^{67}$ Cfr. par. 6 supra.

${ }^{68}$ Cfr. par. 4 supra.

${ }^{69}$ Sia permesso rinviare a B. CoRTESE, "Sui rapporti tra regolamento Bruxelles I, sistemi nazionali e convenzione di Lugano nell'ottica delle relazioni esterne. Considerazioni critiche a margine del parere 1/03 e della recente giurisprudenza comunitaria", Il diritto dell'Unione europea, 2008, pp. 533-574; sempre nell'ottica di una critica all'impostazione seguita dalla Corte si veda P. FrANZINA, "Le condizioni di applicabilità del regolamento (CE) n. 44/2001 alla luce del parere 1/03 della Corte di giustizia", Rivista di diritto internazionale, 2006, pp. 948-976, specie p. 972 ss. Sul parere si vedano inoltre N. LaVRanos, "Opinion 1/03, Lugano Convention", Common Market Law Review, Vol. 43, 2006, pp. 1087-1100; E. Cannizzaro, "Le relazioni esterne della Comunità: verso un paradigma unitario?", Il diritto dell'Unione europea, 2007, pp. 223-238; L.S. Rossi, "Conclusione di accordi internazionali e coerenza del sistema: l'esclusività della competenza comunitaria," Rivista di diritto internazionale, 2007, pp. 1008-1027. Sugli sviluppi della giurisprudenza della Corte circa la dimensione esterna della competenza dell'Unione in materia di diritto internazionale privato nel parere $1 / 13$ si vedano gli studi contenuti in P. FranzinA, Ed., The External Dimension of EU Private International Law after Opinion 1/13, Intersentia, Cambridge, 2017, pp. 1-226.
} 
parere 1/03, la Convenzione del 2007 è stata conclusa esclusivamente dall'Unione e dunque si applica ai suoi Stati membri in quanto tali.

Con l'uscita del Regno Unito dall'Unione si aprono dunque due scenari per una sua possibile adesione successiva a titolo individuale a quella convenzione.

Il primo è quello un'adesione basata su semplice notifica della volontà unilateralmente espressa dallo Stato aderente: questo meccanismo è però riservato agli Stati che divengano parte dell'Associazione Europea di Libero Scambio AELS/EFTA ${ }^{70}$. Per il Regno Unito la cosa appare oltremodo improbabile, per le ragioni politiche che hanno portato alla Brexit: adesione AELS/EFTA e ...take back control sono scelte in totale contraddizione ${ }^{71}$.

Il secondo scenario è quello di un'adesione come Stato non membro né dell'UE né di AELS/ EFTA: in tal caso, però, la Convenzione prevede che l'adesione sia subordinata al consenso di tutte le parti contraenti ${ }^{72}$. Tuttavia, né il Regno Unito, né l'UE, né gli Stati EFTA sembrano essersi dati alcuna particolare premura, ad oggi, per garantire una veloce adesione del Regno Unito a quella convenzione, come invece avrebbe consentito la previsione dell'art. 129, par. 4, dell'Accordo di recesso. Vi è unicamente una serie di dichiarazioni prodotte dagli Stati membri AELS/EFTA, a sostegno dell'intenzione del Regno Unito di aderire a tale convenzione ${ }^{73}$, ma quelle dichiarazioni non sono neppure pienamente concordi: laddove Norvegia e Islanda hanno dichiarato che accolgono positivamente l'intenzione del Regno Unito di aderirvi e che sosterranno tale richiesta, la Svizzera si è limitata alla prima, meno impegnativa, dichiarazione. Qualsiasi anticipazione della posizione negoziale dell'Unione, in merito, sarebbe prematura: è però d'obbligo la prudenza se, come è il caso, un accordo sul punto si sarebbe già potuto negoziare, e non lo si è fatto.

11. Fino al recesso, il Regno Unito era vincolato, in quanto Stato membro dell'Unione europea, dalla Convenzione dell'Aja del 2005 sugli accordi di scelta del foro ${ }^{74}$. In previsione del recesso, il Regno Unito aveva depositato, il 28 dicembre 2018, uno strumento di accessione a quella convenzione, insieme ad una nota verbale e alcune dichiarazioni, presentate nei mesi seguenti. Tale strumento, con i relativi accessori, è stato ritirato il 31 gennaio 2020, in considerazione dell'Accordo di recesso e dell'istituzione, con quello, di un periodo transitorio durante il quale la Convenzione continuerà ad applicarsi al Regno Unito in quanto diritto dell'Unione. Nella relativa dichiarazione di ritiro dello strumento di accessione si anticipa tuttavia l'intenzione di depositare un nuovo strumento di accessione "at the appropriate time prior to the termination of the transition period" 75 .

La convenzione del 2005, seppur non di amplissima portata ${ }^{76}$ tanto per il suo oggetto limitato ad accordi di giurisdizione esclusiva ${ }^{77}$, quanto per l'ampiezza delle situazioni e materie escluse dal suo campo di applicazione ${ }^{78}$, quanto infine per l'esistenza di limiti generali nel suo regime ${ }^{79}$, costituirà nei

\footnotetext{
${ }^{70}$ Convenzione di Lugano (2007), art. 71.

${ }^{71}$ Appartiene ad... un'era lontana, in termini di evoluzione tecnologica, ma colpisce bene il segno la definizione del sistema EFTA/AELS come "fax democracy": se ne veda una delle più note apparizioni nel dibattito pubblico europeo nell'editoriale a firma Charlemagne apparso su The Economist del 7 ottobre 2004, accessibile anche oggi in internet su https://www.economist. com/europe/2004/10/07/the-norwegian-option (visto in luglio 2020).

${ }^{72}$ Convenzione di Lugano (2007), art. 72.

${ }^{73} \mathrm{Si}$ veda la nota stampa del Ministry of Justice del 28 gennaio 2020, facente riferimento alle dichiarazioni adottate dagli Stati EFTA in quello stesso giorno, a margine della conclusione dell'accordo sulle conseguenze della separazione: https://www. gov.uk/government/news/support-for-the-uks-intent-to-accede-to-the-lugano-convention-2007 (vista in luglio 2020)

${ }^{74}$ Convention of 30 June 2005 on Choice of Court Agreements. Sulla convenzione v. in primis T. HARTLEY e M. DogauCHI, Explanatory Report on the 2005 Hague Choice of Court Agreements Convention, accessibile on-line all'indirizzo https://assets. hcch.net/docs/0de60e2f-e002-408e-98a7-5638e1ebac65.pdf; v. inoltre C. KesSEDJIAN, "La Convention de La Haye du 30 juin 2005 sur l'élection de for", Clunet, 2006, pp. 813-850; L. Usunier, "La Convention de La Haye du 30 juin 2005 sur les accords d'élection de for. Beaucoup de bruit pour rien?", Revue critique de droit international privé, 2010, pp. 37-81.

${ }^{75}$ Tutte le dichiarazioni citate nel testo sono accessibili al sito della Conferenza dell'Aja di Diritto Internazionale Privato, alla pagina https://www.hcch.net/en/instruments/conventions/status-table/notifications/?csid=1318\&disp=resdn (vista in luglio 2020).

${ }^{76} \mathrm{Si}$ vedano in tal senso le osservazioni di A. Briggs, "Brexit...," cit., p. 274 s.

${ }^{77}$ Convenzione dell'Aja del 2005, artt. 1 e 3.

${ }^{78}$ Convenzione dell'Aja del 2005, art. 2.

${ }^{79}$ Penso in particolare alla previsione del suo art. 6, lettera c), secondo cui non si riconosceranno clausole di scelta del foro
} 
rapporti tra il Regno Unito e l'UE uno strumento capace di dare un concreto contributo in termini di prevedibilità delle situazioni giuridiche transnazionali ${ }^{80}$.

Quanto alla Convenzione dell'Aja del 2019 sul riconoscimento e l'esecuzione delle decisioni straniere in materia civile e commerciale, essa non è ancora in vigore, e né il Regno Unito né l'Unione l'hanno ancora accettata. Tuttavia, dato che la sua entrata in vigore presuppone l'accettazione di due sole parti contraenti, essa costituisce un agevole, seppur non ambizioso, quadro di riferimento per un futuro meccanismo di riconoscimento delle sentenze tra l'UE (e suoi Stati membri) e il Regno Unito ${ }^{81}$.

\section{Cliff-edge scenario}

12. Quale lo scenario in caso di mancata proroga del periodo transitorio ed in assenza di un accordo comprensivo raggiunto entro il termine di quel periodo?

Si dovrà distinguere, a tale proposito, il profilo della legge applicabile (regimi Roma I e Roma II), da quello di giurisdizione e riconoscimento delle decisioni giudiziarie (regimi Bruxelles e Lugano).

13. In relazione al profilo relativo alla legge applicabile, due elementi convergenti riducono al minimo - seppur senza escluderle completamente - le conseguenze di un mancato accordo, assicurando una tendenziale continuità delle regole giuridiche applicabili in questa materia.

Anzitutto, la dimensione universale dei regimi internazionalprivatistici previsti dai due Regolamenti Roma I e Roma II limita fortemente l'impatto della Brexit sul diritto applicabile davanti ai giudici degli Stati membri dell'Unione, che quei regolamenti continueranno ad applicare.

Ulteriormente, la tendenziale continuità del regime internazionalistico applicabile è garantita dalla scelta del Regno Unito di rendere applicabili, a titolo di retained legislation ${ }^{82}$, i due regolamenti Roma I e Roma II (o, più correttamente, regole nazionali di conflitto costruite tramite rinvio unilaterale al regime internazionalprivatistico posto da quei due regolamenti), anche in assenza di un accordo comprensivo che, al termine del periodo transitorio, assicuri una continuità di relazioni giuridiche tra il Regno e la UE ${ }^{83}$.

Non mancano tuttavia alcuni aspetti in relazione ai quali l'uniformità del regime internazionalprivatistico di qua e di là dalla Manica è destinata comunque ad incrinarsi.

Anzitutto è evidente che, anche in caso di applicazione da parte dei giudici britannici dei regimi Roma I e Roma II a titolo di retained legislation, non opereranno quelle rade previsioni regolamentari che danno specifico rilievo al fatto che il diritto applicabile sia quello di uno Stato membro ${ }^{84}$.

Ancora, qualora la controversia fosse portata davanti ad un giudice di uno Stato membro, si avrà un sostanziale ridimensionamento della possibilità di scegliere uno dei diritti in vigore nel Regno Unito, ove tutti gli elementi della fattispecie in questione siano ubicati in uno o più Stati membri, non potendo in quei casi il richiamo al diritto di uno Stato terzo pregiudicare "l'applicazione delle disposizioni di

qualora "giving effect to the agreement would lead to a manifest injustice". Si veda sul punto J. LANDBRECHT, "The Concepts of "Injustice" and "Public Policy" in Article 6(c) of the Hague Choice of Court Convention", Journal of Private International Law, Vol. 15, 2019, pp. 339-364.

${ }^{80}$ Seppur non senza impedire il risorgere di possibili contrasti in caso di azione intentata in un foro diverso da quello contemplato nella clausola, compresa l'eventualità, in tal caso, di anti-suit injunctions "contro" le corti dell'Unione: cfr. M. AHMED, P. Beaumont, "Exclusive Choice of Court Agreements: Some Issues on the Hague Convention on Choice of Court Agreements and its Relationship with the Brussels I Recast Especially Anti-Suit Injunctions, Concurrent Proceedings and the Implications of BREXIT", Journal of Private International Law, Vol. 13, 2017, pp. 386-410, p. 402 ss.

${ }^{81}$ V. le osservazioni di C. E. Tuo, "The Consequences of Brexit for Recognition and Enforcements of Judgments in Civil and Commercial Matters: Some Remarks", Rivista di diritto internazionale privato e processuale, 2019, pp. 302-318, p. 317. Si veda inoltre una discussione più estesa del regime predisposto dalla convenzione infra al par. 20.

82 The Law Applicable to Contractual Obligations and Non-Contractual Obligations (Amendment etc.) (EU Exit) Regulations 2019, UK Statutory Instrument 2019 No. 834.

${ }^{83}$ UK Statutory Instrument 2019 No. 834, cit., Section 10 e Section 11.

${ }^{84}$ Se ne veda sopra un veloce richiamo al par. 6 del presente scritto. 
diritto comunitario, se del caso, come applicate nello Stato membro del foro, alle quali non è permesso derogare convenzionalmente" ${ }^{85}$.

Inoltre, in mancanza di un accordo che assicuri una qualche forma di coordinamento evolutivo tra i due regimi internazionalprivatistico (UE e britannico), è da attendersi che principi di diritto enunciati dalla Corte di giustizia in tempi successivi alla fine del periodo transitorio possano non trovare accoglimento da parte dei giudici britannici, determinando un lento "allontanamento" del quadro ermeneutico rilevante.

Infine, ed a più forte ragione, eventuali modifiche introdotte successivamente in quei regolamenti dal legislatore dell'Unione non saranno introdotte, se non per separata decisione del legislatore britannico, in quella retained legislation.

Sempre in relazione al diritto applicabile, anche in un cliff-edge scenario si dovrebbero ritenere ancora vigenti per il Regno Unito, in mancanza di una denuncia, la Convenzione di Roma e i suoi due Protocolli, limitatamente ai profili intertemporali e di applicazione territoriale extraeuropea che si sono già analizzati ${ }^{86}$.

Proprio il fatto che in quel contesto sia mantenuta una seppur marginale (perché non obbligatoria e perché limitata ai rinvii proposti da giudici di appello e di ultima istanza) competenza della Corte di giustizia fa tuttavia pensare che il mantenimento in vigore dei due Protocolli sia il frutto di una dimenticanza nel convulso e a tratti disorganizzato atteggiamento negoziale che ha caratterizzato la parte britannica nel processo messo in moto dalla dichiarazione ex art. 50 TUE. Cosicché, una volta la questione emersa, è verosimile attendersi una denuncia espressa di quegli strumenti convenzionali ovvero, in ogni caso, una sostanziale inattuazione da parte dei giudici britannici del potere di rinvio che quei Protocolli istituiscono.

14. Ben diversa è la situazione relativa ai regimi di Bruxelles e Lugano e più in generale in materia di giurisdizione e riconoscimento delle sentenze. In tale ambito, infatti, la natura di convention double degli strumenti sinora applicabili ne limita l'operatività ai rapporti tra Stati contraenti/membri, in una chiave di necessaria reciprocità. Questa andrà inevitabilmente persa con l'uscita del Regno Unito dall'Unione e la fine del periodo transitorio previsto dall'Accordo di recesso, senza che un'eventuale - auspicabile, ma pur sempre improbabile - riproduzione dei titoli di giurisdizione del Regolamento Bruxelles I(bis) a titolo di retained legislation ${ }^{87}$ possa cambiare il risultato in maniera decisiva.

Ora, per raggiungere un risultato di tendenziale stabilità delle relazioni giuridiche in ambito civile e commerciale prima e dopo la Brexit, è richiesto il ristabilimento di un quadro normativo relativo tanto all'esercizio e coordinamento della giurisdizione, quanto al riconoscimento, sulla falsariga dei regimi di Bruxelles e Lugano.

In assenza di un regime di convention double applicabile ai rapporti tra l'Unione e il Regno Unito, si passerebbe all'applicazione dei regimi nazionali di riconoscimento delle sentenze straniere, sia nel Regno Unito, sia nei singoli Stati membri dell'UE.

Contro una tale evenienza non potrebbero opporsi né la reviviscenza di accordi bilaterali da tempo sostituiti dal regime comunitario e poi unionale ${ }^{88}$, né la conclusione di nuovi accordi bilaterali tra il Regno Unito e i singoli Stati membri.

\footnotetext{
${ }^{85}$ Regolamento Roma I, art. 3, par. 4, cfr. anche supra par. 6. Si noti che tale limitazione della possibilità di scelta è stata espressamente mantenuta anche nelle disposizioni regolamentari del Regno Unito che determinano la perdurante applicazione del Regolamento quale retained legislation: UK Statutory Instrument 2019 No. 834, cit., Section 10, par. 2 b) e par. 4. Tali previsioni operano a garanzia della perdurante applicazione delle disposizioni di diritto UE intese come non derogabili e mantenute a loro volta nel Regno Unito a titolo di retained legislation: cfr. A. Dickinson, “A View from the Edge”, Oxford Legal Studies Research Paper No. 25/2019, pp. 1-12, a p. 3. Il paper è accessibile on-line nel database SSRN all'indirizzo https://papers.ssrn. $\mathrm{com} /$ sol3/papers.cfm?abstract_id=3356549. Quanto alla possibilità che la portata dell'art. 3, par. 4, del Regolamento Roma I sia temperata dal mantenimento di previsioni (sostanziali) UE a titolo di retained legislation cfr. le interessanti osservazioni di $\mathrm{P}$. Bertoli, op. cit., p. $620 \mathrm{~s}$.

${ }^{86}$ Supra, par. 7.

${ }^{87} \mathrm{Si}$ veda la discussione di questa ipotesi infra al par. 21 di questo lavoro.

${ }^{88}$ Sostenuta invero senza particolare argomentazione da A. BRIGGS, "Brexit...", cit., p. 272, nonché, con maggiori sviluppi argomentativi, da P. Bertoli, op. cit., p. 626, con argomenti che però non appaiono persuasivi, al di là del formalismo interpretativo su cui sono costruiti, soprattutto alla luce delle considerazioni svolte poco oltre nel testo.
} 
Entrambi questi scenari sembrano incompatibili con la conclusione, raggiunta dalla Corte di giustizia nel già menzionato parere $1 / 03$, circa la competenza esclusiva dell'Unione a concludere accordi con Stati terzi in questi ambiti ${ }^{89}$.

Per la conclusione di nuovi accordi, ça va sans dire. Per la reviviscenza degli accordi bilaterali precedentemente conclusi, a me sembra che ciò non possa avvenire senza un rinnovarsi della volontà pattizia da parte di entrambi i contraenti. Il fatto che un atto dell'Unione ne abbia disposto la sostituzione, lasciandole in vigore solo per limitati profili materiali ed intertemporali ${ }^{90}$ equivale, sul piano del diritto internazionale generale dei trattati, ad una modifica di quelle stesse convenzioni nel senso disposto dal Regolamento. Per estenderne nuovamente la portata occorrerebbe allora una nuova volontà pattizia internazionale. Ma ciò non potrebbe infine portare ad alcun risultato pratico, davanti ai giudici degli Stati membri, data la prevalenza comunque assicurata alle previsioni del Regolamento su un eventuale accordo concluso in violazione dello stesso.

Il ritorno ai principi e alle regole giuridiche pre-convenzionali nel Regno Unito e negli Stati membri dell'Unione (o più in generale nell'Europa continentale) determinerebbe un doppio "fallimento". Si avrebbe, da un lato, il risorgere di conflitti positivi di giurisdizione, con la conseguente incertezza delle relative situazioni giuridiche; dall'altro lato, un'accresciuta difficoltà nella circolazione delle decisioni giudiziarie across the Channel.

15. Ove al termine del periodo transitorio non fosse applicabile - nei rapporti UE Regno Unito - una convention double modellata sui regimi di Bruxelles e Lugano si produrrebbero numerose importanti modifiche nell'approccio britannico, capaci di generare conflitti di giurisdizione.

A mutare sarebbero gli stessi principi informatori del sistema.

Davanti ai giudici britannici risorgerebbe il principio di common law secondo cui l'esercizio della giurisdizione inglese si basa per l'essenziale sulla notifica del $w{ }^{2} t^{91}$, salva l'eccezionale statuizione di forum non conveniens ${ }^{92}$, espressione di una concezione del tutto unilaterale della giurisdizione lontana anni luce dal contesto di coordinamento e fiducia reciproca instaurato ormai da decenni in Europa. Già questo porterebbe ad un marcato disallineamento tra la prassi continentale e quella inglese ${ }^{93}$.

Sempre nell'ottica dell'unilateralismo che connota in materia la common law inglese, non solamente sparirebbero i limiti posti in generale dalla Corte all'esercizio indiscriminato della giurisdizione cautelare ${ }^{94}$, ma tornerebbe attuale il potere dei giudici inglesi di pronunciare anti-suit injunctions

\footnotetext{
${ }^{89}$ V. anche C. E. Tuo, op. cit., p. 308 s.

${ }^{90} \mathrm{Si}$ vedano in particolare gli artt. 69 e 70 del Regolamento Bruxelles I(bis) per quanto riguarda le convenzioni concluse tra il Regno Unito e altri Stati membri "relative alle stesse materie soggette al presente regolamento", di cui si dispone la sostituzione con il Regolamento (art. 69), salva la loro perdurante applicazione alle materie cui il regolamento non si applica e alle "decisioni emesse, agli atti pubblici formalmente redatti o registrati e alle transazioni giudiziarie approvate o concluse prima della data di entrata in vigore del regolamento (CE) n. 44/2001" (art. 70).

${ }^{91}$ Cfr. su punto ancora A. Briggs, "Brexit...”, cit., p. 264 ss.; L. Merret, "La reconnaissance et l'exécution en Angleterre des jugements venant des États de l'Union européenne, post Brexit", Revue critique de droit international privé, 2019, pp. 385-404, p. 390 s. Si veda inoltre, per una discussion estesa dei casi di "service-out jurisdition", nei quali cioè la giurisdizione si basa sulla comunicazione del writ ad un soggetto non presente nel Regno, A. ArZANDEH, "Gateways" within the Civil Procedure Rules and the Future of Service-out Jurisdiction in England", Journal of Private International Law, Vol. 15, 2019, pp. 516-540.

${ }^{92}$ A. Briggs, "Brexit...", cit. p. 268. Si noti che il test per considerare la giurisdizione inglese come non conveniens, è la prova, da parte del defendant, del fatto che esiste un altro foro "which prima facie is clearly more appropriate": si veda in tal senso il dictum contenuto nell'opinion di Lord Goff of Chieveley in quello che è tuttora il leading case in quest'ambito, Spiliada Maritime Corp v Cansulex Ltd [1986] UKHL 10, [1987] AC 460, p. 478. Il testo della sentenza è accessibile all'indirizzo https://www.bailii.org/uk/cases/UKHL/1986/10.html (visto in luglio 2020). Per una discussione più approfondita delle condizioni alle quali può essere concessa la sospensione dell'esercizio della giurisdizione inglese, in particolare alla luce della dottrina del forum non conveniens, v. A. BrigGs, Civil Jurisdiction and Judgments, Routledge, Abingdon, 6th Ed., 2015, p. 398 ss.

${ }^{93}$ Nonché scozzese: sull'origine scozzese dell'istituto del forum non conveniens v. p.e. L. Collins, (Gen. Ed.), Dicey and Morris on the Conflict of Laws, 13. Ed., London, Sweet \& Maxwell, 2000, Vol I, p. 389; più estesamente A. ArZANDEH, "The Origins of the Scottish forum non conveniens Doctrine", Journal of Private International Law, Vol. 13, 2017, pp. 130-151.

${ }^{94} \mathrm{Il}$ riferimento è anzitutto alla sentenza Van Uden Maritime, cit.: l'eclissarsi di quei limiti, al risorgere del common law, è citato tra le conseguenze positive della Brexit da A. BrIGGS, "Brexit...", cit., p. 268.
} 
"contro" l'esercizio di rimedi giurisdizionali davanti a giudici UE e a difesa della propria competenza, negandosi così in radice i principi di coordinamento e di fiducia reciproca insiti nel sistema continentale. Sia chiaro: non si pretende certo qui di contraddire chi, dall'alto di una profonda conoscenza del diritto inglese, sottolinea la natura in personam delle anti-suit injunctions, che non sarebbero dunque in diritto espressione di un ordine rivolto ad un giudice straniero ${ }^{95}$. Il fatto vero è, però, che si tratta di un puro sofisma: il senso di quelle ingiunzioni rimane profondamente unilaterale, poiché rifiuta una prospettiva di pari ordinazione e coordinamento tra le diverse giurisdizioni europee e presuppone invece un'inerente preminenza della determinazione di esercizio della giurisdizione nazionale (nella specie, di una delle entità che compongono il Regno Unito), quale che sia la determinazione in diritto inglese del destinatario dell'ordine espresso nella anti-suit injunction.

Così come unilaterale è la policy di non considerare la lis alibi pendens quale ragione necessaria di astensione dall'esercizio della giurisdizione del Regno Unito ${ }^{96}$, gestendo le ipotesi di cause parallele tramite una valutazione discrezionale di appropriatezza del foro. Ciò perché, tra l'altro, si tratta di una valutazione basata su una tendenziale preferenza del foro inglese su quello straniero ${ }^{97}-\mathrm{a}$ dispetto della una retorica di understatement ed equidistanza utilizzata per giustificare il rifiuto del principio continentale ${ }^{98}$.

16. Più nel dettaglio, con il riapparire dell'impostazione di common law si avrebbe in concreto una significativa divaricazione dei titoli di giurisdizione applicabili nel Regno Unito e nell'Unione. Ciò, unito al rovesciamento dei principi cardine del sistema, non più orientato alla certezza ma ad una (unilaterale concezione di) giustizia internazionalprivatistica del caso concreto, darebbe luogo ad una situazione ampiamente meno prevedibile dell'attuale.

Qui di seguito si dà brevemente conto di alcune di queste modifiche, prima in riferimento al common law inglese, poi in relazione agli approcci continentali.

Quanto al Regno Unito, in primo luogo non è chiaro in che misura un'azione iniziata nel Regno Unito contro un convenuto persona fisica domiciliata in quello Stato potrà ritenersi compresa nell'ambito del (vasto) potere delle corti britanniche basato su un service within the jurisdiction. A seconda del prevalere di un'interpretazione restrittiva o di una invece ampia del criterio della physical presence potrebbero darsi casi in cui il domicilio non sia considerato sufficiente a incardinare la giurisdizione, laddove il convenuto possa dimostrare che al momento della consegna del writ si trovava concretamente all'estero ${ }^{99}$.

${ }^{95}$ A. BRIGGS, "Brexit...”, cit., p. 272, per l'affermazione citata nel testo; si veda inoltre ivi alle pp. 270 e s. la dotta disamina dell'istituto in diritto inglese.

${ }^{96}$ A. BRigGs, "Brexit...", cit., p. 268. Se ne veda anche la trattazione nel contesto del più ampio del problema del contenzioso parallelo internazionale e transnazionale in C. McLACHLAN, "Lis Pendens in International Litigation", Recueil des cours de l'Academie de droit international privé de La Haye, Vol. 336, Nijhoff, Leiden 2008, pp. 199-554, anche nella ristampa separata per Nijhoff del 2009, 467 pp., specie al cap. II (p. 91 ss. e seguenti della ristampa separata).

${ }^{97}$ Si ponga mente al cd. test Spiliada, cui s'è fatto cenno alla nota 92 supra, ed in particolare al fatto che, da un lato, la concessione di uno staying order è sostanzialmente esclusa in caso di scelta del foro inglese (anche laddove l'esistenza di quella scelta sia contestata da una parte), mentre dall'altro lato la tendenziale applicabilità della legge inglese viene essa stessa intesa come elemento di forte ostacolo alla concedibilità di un tale order: cfr. A. BRIGGS, Civil Jurisdiction and Judgments, cit., p. $398,408$.

${ }^{98}$ Si rimanda qui all'autorevole dictum di Lord Goff of Chieveley in Airbus Industrie GIE v Patel, [1999] 1 AC 119 (HL), in cui, dopo aver descritto la soluzione continentale (allora comunitaria) come capace di evitare "clashes between jurisdictions" degli Stati membri, ma al prezzo di una rigidità che può generare injustice, si descrive l'approccio di common law come basato sull'assunto, più neutrale, dell'esistenza di "a jungle of separate, broadly based, jurisdictions all over the world". Dato che ci si muove in una giungla di giurisdizioni indipendenti e non in un contesto necessariamente coordinato, è allora piuttosto naturale che "parallel proceedings in different jurisdictions are not of themselves regarded as unacceptable", che tanto vi sono a disposizione le strade, alternative, della sospensione del giudizio, o dell'adozione di una anti-suit injunction. La concessione di quest'ultima sarà piuttosto naturale ove la corte inglese ritenga che "England is the natural forum for the resolution of the dispute". Il testo della sentenza è accessibile via internet alla pagina https://publications.parliament.uk/pa/ld199798/ldjudgmt/ jd980402/patel01.htm (vista in luglio 2020). Si colleghi il tutto al test per ritenere che il foro inglese, nonostante la consegna del writ, è forum non conveniens (supra, note 92 e 97) e si avrà il quadro completo.

${ }^{99}$ Nonostante l'auspicio che prevalga l'interpretazione di equivalenza del domicilio, come legame giuridico fattuale di durata, alla presenza come legame fattuale puntuale: per tale auspicio cfr. L. Merret, op.cit., p. 390. 
In secondo luogo, in relazione ad azioni intentate contro persone giuridiche, l'elemento del place of incorporation, attualmente sufficiente a fondare il domicilio della persona giuridica nel Regno Unito $^{100}$, perderà tale rilievo di fronte ai giudici inglesi ${ }^{101}$.

In terzo luogo, nel vigore dei principi di common law si avrà un notevole ampliamento della giurisdizione sul co-defendant rispetto alle previsioni dell'attuale art. 8, par. 1, Regolamento Bruxelles I(bis) ${ }^{102}$. In quarto luogo, non avranno più automatico rilievo davanti ai giudici britannici le competenze imperative o esclusive previste dai regimi di Bruxelles e Lugano. In particolare, in relazione alle ultime, si può qui citare la significativa differenza tra la giurisdizione esclusiva prevista ora dall'art. 24 del Regolamento Bruxelles I(bis) - estesa non solo ai diritti reali immobiliari ma anche ai contratti di locazione di immobili - e le eccezioni alla cd. Moçambique rule di common law, che includono nella subject matter jurisdiction del giudice inglese anche pretese contrattuali relative a immobili situati fuori dal Regno ${ }^{103}$.

Ancora, si possono segnalare qui l'incertezza sul regime applicabile davanti alle giurisdizioni UE in caso di clausole di scelta del foro a favore dei giudici britannici ${ }^{104}$ e l'ampliamento della giurisdizione al co-defendant rispetto alle previsioni dell'attuale art. 8, par. 1, Regolamento Bruxelles I(bis) ${ }^{105}$, ma l'elenco potrebbe continuare ${ }^{106} \ldots$

Parallelamente, anche davanti ai giudici degli Stati membri dell'Unione le regole applicabili a controversie in situazioni che coinvolgono il Regno Unito si modificherebbero in misura significativa.

Anzitutto, tornerebbero ad applicarsi, in molti Stati membri, regole nazionali di giurisdizione contenenti titoli esorbitanti: si pensi, a titolo di esempio, al Vermögensgerichtsstand previsto dal $\S 23$ ZPO o al forum actoris a beneficio del cittadino francese previsto dall'art. 14 code civil.

Inoltre, anche il principio della lis pendens risulterebbe depotenziato: in caso di parallela pendenza di un giudizio davanti alle corti britanniche, infatti, i giudici degli Stati membri dell'Unione non sarebbero più chiamati ad applicare l'art. 31 del Regolamento Bruxelles I(bis), ma il meno esigente art. 33 , in collegamento con l'art. 34, per l'ipotesi di cause connesse ${ }^{107}$.

17. Venendo al riconoscimento delle decisioni, l'uscita del Regno Unito tanto dal regime di Bruxelles quanto da quello di Lugano comporterà un radicale cambio di regime.

Ora, una voce molto autorevole nella dottrina inglese ha chiaramente affermato che, al di là del cambio formale di regole applicabili, non vi sarebbero in realtà importanti conseguenze nella vita reale, sostenendo in buona sostanza che, data l'importanza della "piazza" di Londra nel commercio internazionale, nessun serio attore commerciale si potrebbe comunque permettere il lusso di ignorare una decisione della Commercial Court ${ }^{108}$, con il che sarebbe garantita de facto la circolazione di quelle decisioni.

Una posizione del genere non sembra però condivisibile, non perché non sia vera in una qualche misura la constatazione di fondo circa l'esistenza di mores mercatorum ${ }^{109}$ legati anche a specifiche "piazze" commerciali, ma perché è troppo limitata la visuale che si è in tal modo scelta.

\footnotetext{
${ }^{100}$ Art. 63, par. 2, Regolamento Bruxelles I(bis).

${ }^{101} \mathrm{Si}$ argomenti dalla decisione della Court of Appeal in Adam v Cape Industries [1990] Ch 433. Cfr. ancora L. MerReT, op. cit., p. 390 ss., con riferimenti nelle note alla dottrina inglese prevalente in materia.

${ }^{102}$ Cfr. A. Briggs, "Brexit...," cit., p. 267.

${ }^{103}$ Cfr. A. Briggs, Civil Jurisdiction and Judgments, cit., pp. 383, 386.

${ }^{104}$ Cfr. G. RüHL, "Judicial Cooperation in Civil and Commercial Matters After Brexit: Which Way Forward?", International and Comparative Law Quarterly, Vol. 67, 2018, pp 99-128, p. 113; A.R.E. KISTLER, "Effect of Exclusive Choice-of-Court Agreements in Favour of Third States within the Brussels I Regulation Recast", Journal of Private International Law, Vol. 14, No. 1, 2018, pp. 66-95.

${ }^{105}$ Cfr. A. BRigGs, "Brexit...”, cit., p. 267.

${ }^{106}$ Segnalando ad esempio la tendenza a trasformare una scelta di legge in una clausola di elezione del foro inglese: cfr. M. Hоoк, "The Choice of Law Agreement as a Reason for Exercising Jurisdiction", International and Comparative Law Quarterly, vol 63, 2014, pp. 963-975, con riferimento in particolare alla decisione della High Court in Navig8 Pte Ltd v Al-Riyadh Co ('The Lucky Lady') [2013] EWHC 328, [2013] 2 Lloyd's Rep 104 (Com Ct).

${ }^{107}$ Cfr. per tutti P. Franzina, "Lis pendens Involving a Third Country under the Brussels I-bis Regulation: An Overview", Rivista di diritto internazionale privato e processuale, 2014, pp. 23-41.

${ }^{108}$ Cfr. A. Briggs, "Brexit...", cit., p. 273.

${ }^{109}$ Da ultimo si veda la stimolante analisi critica delle teorie della lex mercatoria sviluppata da A.-L.- CALvo CARAVACA, J.
} 
Il fatto è che il diritto internazionale privato dell'Unione relativo alla materia commerciale non si occupa e non si può occupare solo del big business. Anzi, in quell'ambito non è neppure così fondamentale il suo contributo, poiché a garantire la stabilità delle relazioni tra attori abituali di una determinata branca del commercio internazionale c'è già il canale arbitrale e il contesto della c.d. lex mercatoria, in cui va probabilmente iscritta anche l'osservazione della irresistibile cogenza di una decisione giudiziaria resa dalle corti di "major commercial or trading centres".

In relazione all'attività commerciale, il diritto dell'Unione nel suo complesso, e la predisposizione del sistema di Bruxelles/Lugano come strumentario giuridico funzionalmente connesso agli obiettivi del primo, serve invece per l'appunto a garantire il buon funzionamento del mercato interno.

In un contesto siffatto, l'obiettivo è ben più ambizioso. Non si tratta tanto di garantire un quadro in cui i grandi attori del commercio internazionale continuino a scegliere l'una o l'altra piazza commerciale come luogo di soluzione delle loro eventuali controversie: l'obiettivo è quello di una piena integrazione dei mercati dei diversi Stati membri, a beneficio di tutti gli operatori e più in generale come strumento di crescita del benessere collettivo delle rispettive società nazionali.

Nello specifico ambito della disciplina del riconoscimento, l'obiettivo è allora garantire una stabilità di relazioni giuridiche sui cui possano fare affidamento tutti gli operatori commerciali attivi nel mercato interno, anche piccoli e medi, ma anche i soggetti non commerciali che con quelli entrano in contatto: lavoratori, consumatori, contraenti di assicurazione. Ed è sul mantenimento o meno di questo risultato, anche dopo l'uscita del Regno Unito dall'Unione e dal suo mercato interno, che si misurerà l'adeguatezza o meno della risposta istituzionale alle sfide della Brexit.

Ora, il rischio di un profondo fossato tra un prima e un dopo Brexit è, in quest'ambito, assai marcato.

Anzitutto, il disallineamento radicale delle regole di competenza conseguente all'uscita del Regno Unito dal sistema di convention double di Bruxelles/Lugano renderebbe di per sé in molti casi difficile il riconoscimento delle sentenze delle corti del Regno Unito nei Paesi dell'Europa continentale. Ciò perché al riconoscimento delle sentenze britanniche tornerebbero ad applicarsi, nei diversi Stati membri dell'Unione, le regole interne generali relative al riconoscimento delle sentenze di Stati terzi - in assenza, beninteso, di obblighi convenzionali relativi al riconoscimento derivanti o da strumenti bilaterali eventualmente "sopravvissuti" all'adozione del regime regolamentare ${ }^{110}$, o dalla partecipazione dell'Unione e del Regno Unito a strumenti predisposti dalla Conferenza dell'Aja ${ }^{111}$.

Ora, nel caso di riconoscimento secondo le regole nazionali dello Stato richiesto, e limitandoci qui a prendere a riferimento i sistemi di diritto internazionale privato di Italia, Germania, Francia e Spagna, si imporrebbe in ogni caso la verifica della compétence indirecte del giudice britannico la cui decisione si chiede di riconoscere. In tal senso dispongono, come è noto, l'art. 64 della legge 218 del 1995, in Italia, il $\S 328$ della ZPO in Germania, la costante giurisprudenza di cassazione in Francia ${ }^{12} \mathrm{e}$ l'art. 46 della Ley 29/2015 ${ }^{113}$ in Spagna.

L'impostazione, va notato, non è priva di varianti nei diversi sistemi qui menzionati: se in Francia s'impone unicamente la verifica di una connessione "caracterisée" 114 tra la controversia e il foro straniero, in Italia e in Germania si richiede più radicalmente la verifica della c.d. Spiegelzuständigkeit, consistente nel verificare se il giudice straniero sarebbe stato competente secondo i criteri di competenza

Carrascosa GonzÁlez, "Lex Mercatoria and Private International Arbitration”, Cuadernos de Derecho Transnacional, Vol. 12, 2020, pp. 66-85.

${ }^{110}$ Fenomeno che si ritiene di escludere, per le ragioni illustrate supra, par. 14.

${ }^{111}$ Sulla cui solo parziale adeguatezza cfr. le osservazioni svolte infra, par. 20.

${ }^{112}$ Cfr. Cour de Cassation, 1.re Ch. Civ., 20 février 2007, pourvoi no 05-14.082, Bulletin des arrêts des chambres civiles, 2007, I, no 68. La sentenza è commentata da B. ANCEL E H. MuIR WATT in Revue critique de droit international privé, 2007, p. 420.

${ }^{113}$ Ley 29/2015, de 30 de julio, de cooperación jurídica internacional en materia civil, Boletín Oficial del Estado, n.182 del 31 luglio 2015, sez. I, p. 65906 ss.

${ }^{114}$ Cfr. Cour de Cassation, 1.re Ch. Civ., 6 février 1985, pourvoi no 83-11.241, Bulletin des arrêts des chambres civiles, 1985, I, no 55: « Toutes les fois que la règle française de solution des conflits de juridiction n'attribue pas compétence exclusive aux tribunaux français, le tribunal étranger doit être reconnu compétent, si le litige se rattache de manière caractérisée au pays dont le juge a été saisi et si le choix de la juridiction n'a pas été frauduleux ». La sentenza è commentata da Ph. FrancescAKIS in Revue critique de droit international privé, 1985, p. 243 e da A. Huet in Clunet, 1985, p. 460. 
diretta (giurisdizione) vigenti nello Stato richiesto ${ }^{115}$. La soluzione spagnola appare in qualche misura intermedia, in quanto, pur richiedendo il testo normativo in prima battuta soltanto un collegamento "razonable" ${ }^{116}$, si verifica in buona sostanza che la competenza del giudice straniero sia fondata su criteri simili (anche se non necessariamente equivalenti) a quelli utilizzati dal legislatore spagnolo ${ }^{117}$, pur senza escludersi che un tale collegamento ragionevole sussista anche in altre situazioni ${ }^{118}$.

Ora, il parallelo ritorno ad un sistema di affermazione della giurisdizione britannica basato sui principi di common la $w^{119}$ porterebbe in molti casi ad un difetto del requisito della compétence indirecte. Ciò prescinde in larga misura dall'approccio più o meno rigido alla verifica di quel requisito, data la natura tendenzialmente esorbitante, nella sistematica continentale, di alcuni tra i criteri principali di giurisdizione di common law: dalla presenza fisica meramente temporanea del convenuto al momento della consegna del writ, alle condizioni della service out jurisdiction. In questo senso, possono menzionarsi, a titolo di esempio, la conclusione su suolo britannico del contratto di cui si discute, o addirittura il mero fatto che quel contratto sia stato concluso tramite un rappresentante che risieda o abbia attività nel Regno Unito ${ }^{120}$, o ancora l'estrema larghezza con cui si accetta l'estensione della giurisdizione inglese su un co-defendant che possa dirsi proper party to the claim $^{121}$.

18. Ove al termine del periodo transitorio non fosse instaurato un sistema di convention double nelle relazioni tra la UE ed il Regno Unito, anche il riconoscimento nel Regno Unito delle sentenze rese dai giudici degli Stati membri dell'Unione diverrebbe significativamente più difficile, in virtù dei principi di common law rilevanti in materia ${ }^{122}$. Questi non solo richiedono il controllo della compétence indirecte, ma la subordinano a criteri irriducibilmente peculiari al sistema inglese, rimasto paradossalmente isolato dalle evoluzioni che in materia hanno conosciuto negli ultimi decenni altri sistemi di common law. Un isolamento, si noti, dovuto verosimilmente al fatto che quel sistema era ormai entrato nell'orbita del diritto internazionale privato europeo: il che la dice lunga sulla poca lungimiranza di un progetto politico-economico che intendesse sostituire i legami con il continente e l'Unione europea con quelli, oramai molto più attenuati, con il Commonwealth o con una agognata Anglosphere ${ }^{123}$.

Il regresso che comporterebbe in materia di riconoscimento il ritorno al common law è reso evidente dalla necessità di fondare la compétence indirecte del giudice straniero o sulla presenza nel foro del judgment debtor, o sulla sua partecipazione volontaria al procedimento giudiziario. In questo quadro il cambiamento radicale rispetto all'oggi è reso plasticamente dall'insufficienza dei titoli di giurisdizione speciali previsti dal regime di Bruxelles per giustificare la compétence indirecte dei giudici continentali le cui decisioni si volessero riconoscere nel Regno Unito. Si aggiunga l'incertezza circa la sufficienza o meno del criterio principe del regime europeo, il domicilio del convenuto persona fisica, a soddisfare il criterio di "presenza nel foro" previsto dal common law ${ }^{124}$. Si consideri inoltre, nella stessa ottica, la restrizione delle ipotesi in cui la residenza della persona giuridica rileverebbe come "presenza" nel contesto del giudizio di riconoscimento ${ }^{125}$. Basta considerare queste poche aree di disallineamento

${ }^{115}$ Per l'ordinamento italiano il chiaro tenore testuale dell'art. 64, lett. a), legge 218/95 è confermato dall'interpretazione datane dalla giurisprudenza: Cassazione 28 maggio 2004 n. 10378, Rivista di diritto internazionale privato e processuale, 2005 , p. 129 ss.; Cassazione (S.U.), 8 aprile 2011 n. 8038.

116 Art. 46, par. 1, lett. c) della Ley 29/2015, cit.

${ }^{117} \mathrm{Si}$ veda in questo senso F. GASCÓN INCHAUSTI, "Reconocimiento y ejecución de resoluciones judiciales extranjeras en la Ley de Cooperación Jurídica Internacional en materia Civil", Cuadernos de Derecho Transnacional, Vol. 7, 2015, pp. 158-187, p. 182.

${ }^{118}$ Così per P. A. DE Miguel Asensio, "Coordinación de la Ley de Cooperación Jurídica Internacional en materia Civil con la legislación especial”, Revista Española de Derecho Internacional, Vol. 68, 2016, pp. 99-108, p. 105.

${ }^{119} \mathrm{Su}$ cui si veda supra, par. 15, oltre ai cenni qui riportati poco oltre nel testo.

${ }^{120}$ A. ArZANDEH, "Gateways...", cit., p. 524.

${ }^{121}$ A. BRIGGS, "Brexit...", cit., p. 267.

${ }^{122}$ Se ne veda la puntuale illustrazione in L. Merret, op. cit., pp. 385-404.

${ }^{123}$ Cfr. B. Wellings, A. Mycock, Eds., The Anglosphere: Continuity, Dissonance and Location, Oxford, OUP, 2019, 248 pp.

${ }^{124}$ L. MerRet, op. cit., p. 390. Si veda anche supra, par. 16, nota 99 e testo corrispondente.

${ }^{125}$ L. Merret, op. cit., p. 390 ss., con riferimenti nelle note alla dottrina inglese prevalente in materia: si veda anche supra, par. 16 , note 100 e 101 e testo corrispondente. 
dei criteri di giurisdizione, nel contesto della verifica della compétence indirecte, per dare una prima idea del drammatico cambiamento in vista, in caso di mancato accordo per la fine del periodo transitorio. Senza contare, evidentemente, il radicale cambiamento procedurale e la stessa restrizione dell' ambito in cui un riconoscimento di decisioni straniere sarebbe possibile nel Regno Unito ${ }^{126}$.

19. Detto delle perturbazioni cui darebbe luogo un cliff-edge scenario, e della necessità di porvi rimedio, in quale ambito convenzionale dovrebbero però concentrarsi gli sforzi per assicurare un regime di circolazione il più possibile non perturbato rispetto ad oggi?

L'impossibilità di "resuscitare" il regime della Convenzione del $1968^{127}$, esplicitamente sostituita dal regime regolamentare e dalla stessa Convenzione di Lugano del 2007 per ogni profilo diverso da que1lo, ormai marginale, di diritto intertemporale ${ }^{128}$, unita alla inammissibile reviviscenza delle convenzioni bilaterali ${ }^{129}$ ed alla parziale utilità delle convenzioni dell' $\mathrm{Aja}^{130}$, richiede di concentrare gli sforzi su una possibile estensione al Regno Unito della Convenzione di Lugano del 2007, almeno quale tappa intermedia in vista di un (difficile) accordo bilaterale sull'estensione del regime regolamentare al Regno Unito.

Certo, neppure l'adesione del Regno Unito alla Convenzione di Lugano sarebbe capace di ripristinare, a livello di riconoscimento delle decisioni giudiziarie, lo status quo, data la non piena corrispondenza tra il regime convenzionale e quello regolamentare, così come rimarrebbero alti i rischi di un disallineamento dell'interpretazione della convenzione nella prassi continentale e di common law ${ }^{131}$.

Quanto in particolare alle differenze tra regime convenzionale e regime regolamentare, la più macroscopica riguarda certo la necessità dell'exequatur, che permane in quella convenzione, a differenza di quanto oramai previsto dal Regolamento Bruxelles I(bis). Né appare verosimile che, in una fase di revisione che puntasse ad eliminare le altre difformità tra il regime di Bruxelles e quello di Lugano, l'Unione sia disposta a rinunciare, anche nel regime convenzionale, all'exequatur: si tratta infatti di un passaggio strettamente legato all'instaurazione dello spazio di libertà, sicurezza e giustizia, che potrebbe non essere opportuno estendere a chi, come il Regno Unito, ha deciso di lasciare l'Unione proprio in polemica con l'elemento principale di quello spazio, la libera circolazione delle persone.

Allo stesso tempo, non tutte le innovazioni del regime di Bruxelles che sono state introdotte prima nel Regolamento 44/01 e poi nel Regolamento 1215/2012 si ritrovano nel regime predisposto dalla Convenzione di Lugano del 2007 ${ }^{132}$; inoltre, le ulteriori innovazioni che si vorranno introdurre al regime regolamentare con novelle adottate in procedura legislativa ordinaria non troveranno strada nel contesto convenzionale che all'esito di una successiva, incerta revisione, da operarsi secondo i classici meccanismi del diritto interazionale dei trattati.

Ciò nondimeno, allo stato l'adesione del Regno Unito alla Convenzione di Lugano appare, nelle condizioni malauguratamente poste in essere dalla Brexit, l'unica via ragionevolmente praticabile per instaurare rapidamente un quadro di regole ispirate dalla reciprocità che consentano, da un lato, di limitare al

\footnotetext{
${ }^{126}$ Si veda ancora L. MerRet, op. cit., p. 387 s.

${ }^{127}$ Per riferimenti alla dottrina favorevole alla reviviscenza della Convenzione del 1968 cfr. J. Ungerer, "Consequences of Brexit for European Private International Law", European Papers, 2019, pp. 395-407, p. 397. Come correttamente osserva C.E. Tuo, op. cit., 306 ss., del resto, è oramai chiaro che tanto la Commissione nei suoi documenti illustrativi (per quanto formalmente irrilevante sia in tale materia ed in tale contesto procedurale la posizione di quell'Istituzione) quanto sopratutto l'ordinamento del Regno Unito, tramite le Civil Jurisdiction and Judgments (Amendment) (EU Exit) Regulations 2019, non danno alcun rilievo ad una possibile reviviscenza della Convenzione.

${ }^{128}$ Il che spiega per l'appunto perché la convenzione non sia stata formalmente terminata, aspetto su cui invece inutilmente si appuntano le attenzioni di chi la vorrebbe riespandersi ai rapporti tra Stati UE e Regno Unito dopo la Brexit.

${ }^{129} \mathrm{Su}$ cui supra, par. 14.

${ }^{130} \mathrm{Su}$ cui infra, par. 20.

${ }^{131}$ Aspetto giustamente sottolineato da B. Hess, "The Unsuitability of the Lugano Convention (2007) to Serve as a Bridge between the UK and the EU after Brexit", Max Planck Institute Luxembourg for Procedural Law Research Paper Series, $\mathrm{N}^{\circ}$ 2018 (2), p. 7.

${ }_{132}$ A mero titolo di esempio, si considerino le innovazioni in materia di litispendenza, su cui v. da ultimo la decisione della High Court in Mastermelt Ltd v Siegfried Evionnaz SA [2020] EWHC 927 (QB), e quelle in ambito di contratti conclusi da consumatori e di contratto di lavoro. Su questi ultimi cfr. C. VAquero LóPEz, "El concepto de contrato de trabajo en el marco del Convenio de Lugano. Comentario a la Sentencia del TJUE de 11 de abril de 2019, asunto C-603/17, Bosworth y Hurley", Cuadernos de Derecho Transnacional, Vol. 12, 2020, pp. 776-783, p. 778 s.
} 
massimo i conflitti di giurisdizione e, dall'altro lato, di ottenere una circolazione quanto possibile facilitata delle decisioni giudiziarie - tutto ciò a vantaggio non solo degli attori abituali di quelle branche del commercio internazionale su cui la "piazza" di Londra ha, e verosimilmente continuerà ad avere, un'influenza determinante, ma anche e sopratutto a favore di quella miriade di soggetti che, di qua e di là del Canale della Manica, hanno contribuito al successo di quel grande esperimento economico e sociale rappresentato dal mercato interno: piccole e medie imprese, lavoratori, consumatori e altri contraenti deboli.

20. In mancanza di un'adesione alla Convenzione di Lugano non rimarrebbe che puntare sull'adesione tanto da parte UE quanto da parte britannica ai due più recenti strumenti predisposti in materia dalla Conferenza dell'Aja di Diritto Internazionale Privato ${ }^{133}$.

Il punto di partenza, sostanzialmente già acquisito ${ }^{134}$, è l'adesione del Regno Unito alla convenzione del 2005 sugli accordi di scelta esclusiva di foro. Questa risolverà molti problemi - ma non tutti ${ }^{135}$ - in relazione ad un novero però limitato di clausole di scelta del foro: quelle aventi natura esclusiva.

A partire da lì, l'obiettivo minimale di ricostruzione di un regime di riconoscimento affidabile presupporrebbe un passo ulteriore: l'adesione, tanto da parte britannica quanto da parte UE, alla Convenzione dell'Aja del 2019 sul riconoscimento delle decisioni straniere ${ }^{136}$.

Tale obiettivo appare certo possibile, seppur non privo di qualche difficoltà ${ }^{137}$, ma ben al di sotto delle necessità delle due parti.

Ciò, in primo luogo, perché non si tratta di una convention double $e^{138}$, capace di risolvere alla radice i problemi in materia di riconoscimento legati alla radicale difformità di alcuni dei criteri di esercizio della giurisdizione di common law rispetto al regime di Bruxelles / Lugano.

In secondo luogo, quella convenzione condiziona il riconoscimento in una parte contraente delle decisioni straniere rese dalle corti di un'altra parte contraente (c.d. Stato di origine) ad una serie di criteri positivi, inseriti su insistenza delle delegazioni "continentali" 139 , che non sarebbero rispettati in molti casi di esercizio della giurisdizione inglese sulla base dei principi di common law.

Al suo art. 5, infatti, la convenzione subordina il riconoscimento al ricorrere di almeno uno dei seguenti requisiti:

a) la persona nei cui confronti si chiede il riconoscimento della sentenza aveva la propria residenza abituale nello Stato di origine al momento in cui essa ha assunto la posizione di parte processuale;

b) la persona fisica nei cui confronti si chiede il riconoscimento della sentenza aveva nello Stato di origine lo stabilimento principale dell'attività economica da cui origina la domanda processuale - anche in tal caso, il momento rilevante è quello in cut il judgment debtor ha assunto la posizione di parte processuale;

c) la richiesta di riconoscimento è rivolta contro il soggetto che ha proposto la domanda giudiziale principale, in relazione alle statuizioni rese su quella domanda;

${ }^{133}$ Il quadro delle convenzioni dell'Aja, nel contesto della riflessione sulla Brexit, è delineato da H. VAN LoON, "Le Brexit et les conventions de La Haye", Revue critique de droit international privé, 2019, pp. 353-365.

${ }^{134}$ Vedi quanto osservato al par. 11 supra.

${ }^{135}$ Cfr. le osservazioni di A. Briggs, "Brexit...", cit., p. 274 s.

${ }^{136}$ Sulla convenzione dell'Aja del 2019 si veda P. A. Nielsen, "The Hague 2019 Judgments Convention - from failure to success?", Journal of Private International Law, Vol. 16, 2020, pp. 385-404; D. STEWART, "The Hague Conference Adopts a New Convention on the Recognition and Enforcement of Foreign Judgments in Civil or Commercial Matters", American Journal of International Law, Vol. 113, 2019, pp. 772-783.

${ }^{137}$ Ciò in ragione, da un lato, del fatto che non vi è in essa una clausola di prevalenza piena delle regole dell'Unione, ma solo una destinata ad operare in situazioni intra UE e, dall'altro lato, in considerazione della non piena corrispondenza delle regole di compétence indirecte della Convenzione con i regimi di competenze imperative ed esclusive del Regolamento Bruxelles I(bis): si vedano su punto le notazioni relative al progetto di convenzione in P. De Miguel Asensio, G. Cuniberti, P. Franzina, C. Heinze, M. Requejo Isidro, The Hague Conference on Private International Law “Judgments Convention”: Study, Brussels, European Parliament, 2018, p. 23 ss.

${ }^{138}$ Nonostante il progetto iniziale prevedesse invece quella strada: cfr. C. E. Tuo, op. cit., p. 317.

${ }^{139}$ D. STEWART, op. cit., p. 777. 
d) la persona nei cui confronti si chiede il riconoscimento della sentenza aveva, al momento in cui essa ha assunto la posizione di parte processuale, uno stabilimento secondario nello Stato di origine, se la domanda processuale origina dalle attività economiche condotte da quello stabilimento;

e) la persona nei cui confronti si chiede il riconoscimento della sentenza ha prestato il proprio esplicito consenso alla giurisdizione dello Stato di origine (con condizioni speciali in caso di contratti dei consumatori);

f) nel caso di rugelose Einlassung, esclusi i contratti dei consumatori;

g) il luogo di esecuzione dell'esecuzione dedotta in giudizio si trova nello Stato di origine (sono esclusi i contratti dei consumatori);

h) il luogo di situazione dell'immobile si trova nello Stato di origine, in caso di domanda relativa all'affitto/locazione di quell'immobile;

i) la decisione si pronuncia su un'obbligazione contrattuale garantita da un diritto reale su un immobile situato nello Stato di origine;

j) la decisione si pronuncia sulla responsabilità extracontrattuale derivante da morte, lesioni, danneggiamento o distruzione di beni materiali e l'azione od omissione che ha direttamente causato il danno si è verificata nello Stato di origine;

k) la decisione si pronuncia sugli aspetti interni di un trust e lo Stato di origine è indicato nell'atto costitutivo come sede dell'amministrazione, o è oggetto di una clausola attributiva di giurisdizione;

1) in caso di domanda riconvenzionale (con diverse condizioni a seconda che se ne chieda il riconoscimento contro l'autore della domanda principale o contro l'autore della domanda riconvenzionale);

m) in caso di clausola di scelta del foro, esclusi i contratti dei consumatori.

Ora, i requisiti positivi di riconoscimento così delineati, molto chiaramente informati all'approccio continentale (e dell'Unione europea in specie), appaiono destinati a limitare sostanzialmente l'effetto utile di quella convenzione nei rapporti UE/Regno Unito, se non dovesse seguire una coerente riforma del diritto inglese in materia.

Mancando una tale riforma, infatti, si rischierebbe di veder escluse dalla possibilità di un riconoscimento nell'Unione le sentenze rese da corti britanniche sulla base della semplice temporanea presenza della persona fisica nel Regno Unito al momento della consegna del writ, o sulla base di alcuni dei criteri che consentono la c.d. service out jurisdiction sulla base di tenuissimi vincoli con il suolo del Regno Unito ${ }^{140}$.

Al tempo stesso, non sarebbero infrequenti anche i casi di sentenze, pur pronunciate da un tribunale di uno Stato membro sulla base dei criteri enunciati dall'art. 5 della convenzione del 2019, che non potrebbero però riconoscersi per il ricorrere di alcune ipotesi di diniego contemplate dall'art. 7, par. 1, della stessa convenzione. Mi riferisco, da un lato, alla lettera d) e, dall'altro, alla lettera e) di quel paragrafo.

La prima di quelle disposizioni prevede che possa rifiutarsi il riconoscimento di una sentenza resa in dispregio di un accordo di scelta del foro che comporti deroga della giurisdizione dello Stato di origine. Ora, nell'assenza di una radicale innovazione dei principi di common law sul punto, sembra ragionevole assumere che questo motivo di diniego si applichi, nel Regno Unito, anche laddove la giurisdizione dello Stato di origine rientri tra quelle che non sopportano deroga in virtù del Regolamento Bruxelles I(bis).

Il secondo motivo di diniego sopra ricordato riguarda le ipotesi di contrasto con una decisione resa tra le stesse parti nello stato richiesto: anche qui, l'assenza di un ravvicinamento tra l'impostazione di common law e l'impostazione continentale alla giurisdizione renderebbe possibile un forum shopping a favore delle corti inglesi, anche dove sussista nell'Unione un titolo di giurisdizione esclusiva, o sia comunque già stata intentata davanti alle corti di uno Stato membro dell'Unione un'azione basata sul

${ }^{140}$ Si veda quanto osservato supra, al par. 17. 
foro generale del domicilio del convenuto o anche soltanto su una delle competenze alternative previste dall'art. 7 del Regolamento Bruxelles I(bis).

In tutte queste ipotesi, l'esercizio della giurisdizione inglese rimarrebbe condizionato alla sola verifica della consegna del writ, salva l'eccezionale dichiarazione che il foro britannico sia non conveniens. Con l'ulteriore conseguenza pratica di rendere non riconoscibile nel Regno Unito la decisione straniera resa nella stessa causa.

21. A questo punto, appare utile ritornare sull'ipotesi di un inserimento nella legislazione del Regno Unito, quale retained legislation, di regole formulate sulla base del regime comune. Si tratta invero di un'ipotesi non (integralmente) contemplata dal governo britannico allo stato ${ }^{141}$, ma pur sempre immaginabile almeno quale strumento transitorio unilaterale, in caso di stallo nei negoziati al termine del periodo transitorio previsto dall'Accordo di recesso.

A favore della percorribilità del percorso qui ipotizzato depongono del resto i regimi unilateralmente introdotti per via regolamentare nella legislazione britannica nel 2019. Attraverso un apposito Statutory Instrument, infatti, il governo britannico ha introdotto un regime modulato sull'art. 7 del Regolamento Bruxelles I(bis), per quanto riguarda la ripartizione della giurisdizione tra le varie parti costituenti il Regno Unito nei confronti di convenuti domiciliati nel Regno Unito. Con lo stesso strumento si sono introdotti nell'ordinamento del Regno Unito regimi modellati sulle sezioni 4 e 5 del Regolamento in relazione ai contratti conclusi da consumatori domiciliati nel Regno Unito ed ai contratti di lavoro conclusi da lavoratori che ivi prestano abitualmente la loro prestazione di lavoro ${ }^{142}$.

Nella stessa direzione - e con maggior forza - depone altresì il regime transitorio previsto, in via generale, per il riconoscimento delle sentenze rese dai giudici deli Stati UE fino al momento in cui il Regolamento Bruxelles I(bis) cesserà di essere applicabile al Regno Unito ${ }^{143}$.

Quali le conseguenze di un'eventuale adozione unilaterale di regole di giurisdizione modellate sul Regolamento Bruxelles I(bis), da parte del Regno Unito?

Se analizzata da un punto di vista formale, è chiaro che l'ipotizzata adozione unilaterale di un regime modulato su quello comune non potrebbe vincolare in alcun modo gli Stati membri dell'Unione (né gli Stati parte alla Convenzione di Lugano). Tuttavia, indirettamente, una scelta del genere permetterebbe di ridurre al minimo le conseguenze dell'abbandono del sistema unionale e convenzionale da parte del Regno Unito.

Ove fossero inserite nella legislazione del Regno Unito regole sull'esercizio della giurisdizione modulate su quelle del regolamento, almeno in relazione a convenuti domiciliati negli Stati dell'Unione e (in termini di esclusione dell'esercizio della giurisdizione britannica) a fattispecie che diano luogo a competenze esclusive degli Stati membri sulla base del Regolamento, nonché ove fosse ulteriormente previsto il rilievo della lis pendens, ed ove infine si costruisse anche la giurisdizione cautelare in modo coerente con l'impostazione del regolamento, si ridurrebbero significativamente le ipotesi di conflitto di giurisdizione, riducendone al minimo la componente ... di origine britannica.

22. Per aversi totale eliminazione di quei conflitti, evidentemente, analoghe regole dovrebbero trovare applicazione anche davanti ai giudici degli Stati membri dell'Unione.

${ }^{141}$ Cfr. G. RuHL, op. cit., p. 118, in riferimento al policy paper "Providing a cross-border civil judicial cooperation framework - a future partnership paper" pubblicato nel 2017 dallo UK Department for Exiting the European Union, ove si segnala il "need to negotiate and agree a new civil judicial cooperation framework". Ė vero però altresì che, in quello stesso documento, si segnala l'impegno a far sì che quel nuovo quadro "build on the strong foundation of existing cooperation". Il policy paper è accessibile on-line all'indirizzo https:/www.gov.uk/government/publications/providing-a-cross-border-civil-judicial-cooperation-framework-a-future-partnership-paper (visto in luglio 2020).

${ }^{142}$ Civil Jurisdiction and Judgments (Amendment) (EU Exit) Regulations 2019. Se ne veda la discussione in A. DiCKINSON, "A View from the Edge", cit.

${ }^{143}$ Civil Jurisdiction and Judgments (Amendment) (EU Exit) Regulations 2019, Regulation 92. Cfr. A. Dickinson, "A View from the Edge", cit., e L. Valkova, Brexit e diritto internazionale privato e processuale in caso di «no deal», Rivista di diritto internazionale privato e processuale, 2019, pp. 230-246. 
Per l'Italia, ciò avverrebbe per molti aspetti sulla base del regime previsto dalla legge di riforma del diritto internazionale privato del 1995. In tal senso opererebbero infatti, da un lato, l'art. 3, comma 2, della legge, che rende applicabili i titoli speciali di giurisdizione del regime di Bruxelles anche in caso di convenuto non domiciliato in uno Stato membro ${ }^{144}$, dall'altro lato gli articoli 4, 5, 7 e 10 in materia, rispettivamente di deroga della giurisdizione italiana, azioni reali aventi ad oggetto immobili siti all'estero, litispendenza e giurisdizione cautelare. Non si tratta di regole del tutto sovrapponibili a quelle del Regolamento Bruxells I(bis), questo è certo ${ }^{145}$, ma è altrettanto vero che sono ispirate agli stessi principi che animano il sistema comune e, pertanto, riducono di molto il rischio di conflitti di giurisdizione con Stati terzi che si ispirassero, nel definire i limiti della propria giurisdizione, a quello stesso sistema.

Non in tutti gli Stati membri, tuttavia, la risposta sarebbe altrettanto "benevola", potendo di per sé trovare applicazione ai rapporti coinvolgenti il Regno Unito titoli di giurisdizione nazionali c.d. esorbitanti ${ }^{146}$.

La soluzione migliore per garantire un tendenziale parallelismo anche in assenza di accordo, di fronte ad un'adozione britannica dei criteri del regime di Bruxelles quale retained legislation, sarebbe allora l'adozione di uno strumento regolamentare ad hoc, con cui estendere, fino al raggiungimento di un accordo sul regime definitivo, l'applicazione di regole modulate su quelle del Regolamento Bruxelles I(bis), "in situazioni che coinvolgano il Regno Unito", per riprendere il linguaggio utilizzato dall'art. 64 dell'Accordo di recesso ${ }^{147}$. La competenza ad adottare un simile regolamento si fonderebbe senza difficoltà sull'art. 81 , par. 2, lett. c), ai termini del quale possono essere adottate misure volte a garantire, in particolare, "la compatibilità delle regole applicabili negli Stati membri ai conflitti [...] di giurisdizione": non facendo riferimento la lettera in questione ai conflitti di giurisdizione tra Stati membri, non sembra difficile ritenerla estesa anche all'ipotesi di norme che evitino conflitti di giurisdizione tra Stati membri e Stati terzi. Si tratta, da un lato, di un profilo essenziale per assicurare il buon funzionamento del mercato interno, le cui regole sono per parte non secondaria applicabili anche a situazioni connesse con Stati terzi: si pensi alla dimensione della circolazione delle merci provenienti da uno Stato terzo e messe in libera pratica, il cui operare può certo coinvolgere un contenzioso commerciale sottratto, invece, all'applicazione del Regolamento Bruxelles I(bis). Dall'altro lato, è la stessa Corte di giustizia, nella sentenza $\mathrm{Owusu}^{148}$ e nel parere $1 / 03^{149}$, ad aver ricondotto all'ambito di applicazione del Regolamento e alla competenza dell'Unione anche i profili di giurisdizione nelle controversie relative a fattispecie connesse esclusivamente ad uno Stato membro e ad uno o più Stati terzi ${ }^{150}$.

Una volta che si fossero, in tal modo, ravvicinate le previsioni sull'esercizio della giurisdizione, anche il profilo del riconoscimento sarebbe meno complesso, evitandosi in linea di massima i problemi legati al controllo della compétence indirecte.

${ }^{144}$ Per la critica di questa scelta (italiana, ma anche spagnola), in quanto non ispirata ad un criterio di reciprocità, v. però, tra gli altri, F. POCAR, "Révision de Bruxelles I et ordre juridique international: quelle approche uniforme?", Rivista di diritto internazionale privato e processuale, 2011, pp. 591-600, p. 595; ID., "Sulla riforma dell'art. 3 comma 2 della legge n. 218/1995", Rivista di diritto internazionale privato e processuale, 2011, pp. 628-634, p. 633.

${ }^{145}$ Ed è stato giustamente criticato l'immobilismo del legislatore italiano, "poco avvezzo alla "manutenzione" delle leggi ai fini di adeguamento agli obblighi internazionali" da C. CAMPIGLIO, "Legge di diritto internazionale privato e regolamenti europei: tecniche di integrazione", Rivista di diritto internazionale privato e processuale, 2018, pp. 292-317, p. 315. Per un'analisi specifica dei rapporti tra articoli 4 e 7 LDIP e disciplina UE v. F.C. ViLLATA, "Sulla riforma degli articoli 4 e 7 della legge $n$. 218/1995 alla luce della proposta della Commissione di revisione del regolamento Bruxelles I", Rivista di diritto internazionale privato e processuale, 2011, pp. 661-669.

${ }^{146} \mathrm{~V}$. quanto osservato velocemente supra, par. 15 , in fine.

${ }^{147}$ Sul significato da darsi a quel termine si vedano le osservazioni svolte supra al par. 8 .

${ }^{148}$ Sentenza della Corte 1. marzo 2005, causa C-281/02, Owusu, Raccolta, 2005, p. I-1383.

${ }_{149}$ Parere della Corte (seduta plenaria) del 7 febbraio 2006, Competenza della Comunità a concludere la nuova Convenzione di Lugano concernente la competenza giurisdizionale, il riconoscimento e l'esecuzione delle decisioni in materia civile e commerciale, parere 1/03, Raccolta, 2006, p. I-1145

${ }^{150}$ Sentenza $O w u s u$, punti 25-29; parere 1/03, punti 144 e ss., ed in specie i punti 148 e 149. Sia sulla sentenza Owusu, sia sul parere, sia consentito rinviare all'analisi svolta in B. CORTESE, op. cit., pp. 546 ss. 


\section{Conclusioni}

23. L'analisi delle conseguenze della Brexit sul diritto internazionale privato e processuale in materia civile e commerciale mette in luce un esito binario.

Se per il profilo del diritto applicabile alle obbligazioni contrattuali ed extracontrattuali, infatti, nessun particolare scossone è in vista qualunque sia l'esito dei negoziati tra il Regno Unito e l'Unione in vista del termine del periodo transitorio, per quanto invece riguarda il profilo della disciplina della giurisdizione e quello del riconoscimento delle decisioni il panorama giuridico attuale rischia di essere stravolto da un sisma di primaria importanza, in caso di uscita senza adeguato accordo.

Per questi ambiti, in particolare, si è messo in luce come, sia in relazione all'esercizio della giurisdizione, sia (sopratutto) in relazione al riconoscimento, neppure un'estensione unilaterale del regime Bruxelles I(bis) che si volesse attuare dai due lati del Canale della Manica darebbe luogo a soluzioni ottimali. In relazione ad entrambi i profili, dunque, rimarrebbe senz'altro auspicabile un definitivo passaggio ad un regime convenzionale "doppio".

Nella specie, ferma restando la tappa intermedia e realistica consistente nell'adesione del Regno Unito alla Convenzione di Lugano, l'obiettivo ultimo dovrebbe essere la conclusione di un accordo bilaterale UE/Regno Unito comportante l'estensione del regime previsto dal regolamento Bruxelles I(bis) al Regno Unito post Brexit.

In fondo, quello che si sta qui preconizzando, è un accordo sullo stile di quello raggiunto all 'interno dell'Unione, tra l'UE e la Danimarca, per l'applicazione del regime regolamentare ${ }^{151}$, nonostante la non partecipazione di quello Stato membro allo Spazio di Libertà Sicurezza e Giustizia.

Ora, è ben vero che la prospettiva di estendere al Regno Unito il modello dell'Accordo UEDanimarca appare estremamente ambiziosa e politicamente poco digeribile nello scenario britannico attuale- dati gli impegni presenti nell'accordo danese circa la giurisdizione della Corte di giustizia, compresa la possibilità di un procedimento di infrazione ${ }^{152}$.

Tuttavia il percorso di avvicinamento che qui si immagina potrebbe rendere plausibile un accordo del genere senza controllo della Corte, ma con un meccanismo di controllo internazionalistico capace di agganciare, di fatto, l'accordo all'evoluzione del sistema regolamentare, come definito anche dalla giurisprudenza della Corte.

Di quale percorso si tratta?

Le prime due tappe (in verità alternative) sono la naturale conseguenza dell'analisi condotta qui sopra, e sono in effetti già state illustrate nel corso del presente lavoro. Su di esse sarà dunque possibile limitarsi ad un cenno.

Si dovrebbe muovere senz'altro dalla (spedita) conclusione di un accordo di adesione alla Convenzione di Lugano 2007, così come essa è attualmente in vigore con gli Stati EFTA. Ciò garantirebbe una sorta di rete di salvataggio buona ad evitare scenari catastrofici, rendendo negoziazioni ulteriori - e passi unilaterali di buona volontà all'interno di un quadro negoziale - sicuramente più agevoli.

In ogni caso, si dovrebbe procedere, in parallelo nel Regno Unito e nell'Unione, all'introduzione unilaterale di regole di continuità, modellate sul regolamento, in vigore a titolo transitorio o comunque residuale (applicabili, cioè, in mancanza di uno specifico accordo bilaterale o di una convenzione latamente equivalente, come la Convenzione di Lugano). Ciò consentirebbe di mantenere (o ricostituire) la fiducia sul fatto che, in quest' area non si vuole alcuna discontinuità sostanziale - come in altre aree dove scenari del genere erano invero stati predisposti nelle settimane più buie dei negoziati ${ }^{153}$.

${ }^{151}$ Accordo tra l'Unione europea e il Regno di Danimarca concernente la competenza giurisdizionale, il riconoscimento e l'esecuzione delle decisioni in materia civile e commerciale, fatto il 19 ottobre 2005, concluso per l'Unione dal Consiglio con la Decisione 2006/325/CE, del 27 aprile 2006, è in vigore dal 1. luglio 2007. L'Accordo, il cui testo è in GUUE n. L 299 del 16 novembre 2005, è stato da ultimo modificato, per gli aspetti che interessano il presente lavoro, con la notifica danese del 22 aprile 2015 che ne adegua il contenuto al Regolamento Bruxelles I(bis): GUUE n. L 182 del 10 luglio 2015.

${ }^{152}$ Basti pensare al rinvio pregiudiziale previsto dall'art. 6 dell'Accordo UE-Danimarca e al ricorso per infrazione previsto dall'art. 7 dello stesso Accordo. Cfr. R. Aikens, A. Dinsmore, op. cit., pp. 914, 915; G. RüHL, op. cit., p. 120.

${ }^{153} \mathrm{Si}$ vedano le comunicazioni della Commissione sulla cosiddetta no-deal Brexit preparedness, ed in particolare la quarta comunicazione del 12 giugno 2019, $\operatorname{COM}(2019) 276$ final, Stato attuale dei preparativi delle misure di emergenza in vista del recesso del Regno Unito dall'Unione europea. 
A questo punto, sarebbe possibile immaginare la conclusione di un Accordo bilaterale sull'applicazione del regime regolamentare nei rapporti UE / Regno Unito, che sostituisca però alla previsione "danese" di un sostanziale impegno al rispetto della giurisprudenza della Corte di giustizia, accompagnato dall'accesso dello Stato in questione alle procedure davanti Corte aventi ad oggetto il Regolamento e dal potere della Commissione di proporre un ricorso di infrazione davanti alla Corte, un meccanismo differente, di stampo internazionalistico ma capace di ottenere nella sostanza lo stesso risultato.

L'ipotesi che si vuole qui delineare è quella di un organo bilaterale (esecutivo) di controllo dell'applicazione di quell'Accordo, nell'ambito del quale si operi un costante monitoraggio della giurisprudenza della Corte relativa al Regolamento. A quell'organo si dovrebbe attribuire il potere di introdurre, su richiesta di una parte ma con decisione consensuale, le modifiche necessarie all'adeguamento dell'Accordo non solo ad eventuali novelle regolamentari - per le quali si prevedrebbe comunque un coinvolgimento a titolo consultivo del Regno Unito nei lavori del Consiglio, ma le modifiche necessarie all'allineamento dell'Accordo all'evoluzione della giurisprudenza della Corte relativa al Regolamento, ove si ritenga che la giurisprudenza delle corti britanniche, relativa all'applicazione dell'Accordo, non si sia adeguata ai principi di diritto così emersi.

Parallelamente, si dovrebbe prevedere che, mancando il consenso sull'introduzione delle modifiche richieste da una parte (in ipotesi, l'Unione), l'Accordo bilaterale sia terminato.

In tal modo, da un lato, si instaurerebbe un meccanismo capace di garantire il perdurante parallelismo della disciplina del Regolamento e di quella applicabile nei rapporti con il Regno Unito. Dall'altro lato, si eviterebbe una formale sottoposizione del Regno Unito ai... capricci di una Corte di giustizia incapace di comprendere la bontà delle soluzioni di common law ${ }^{154}$. Anche perché, ove il legislatore dell'Unione lo volesse, rimarrebbe comunque aperta la strada di una modifica espressa del regime Regolamentare capace di ricondurre, per il futuro, il parallelismo su binari diversi da quelli definiti dalla Corte ed accettabili dalla controparte britannica: l'esempio del recast in materia di lis pendens è lì a dimostrare che una strada del genere è tutt'altro che teorica.

In un sistema del genere l'incentivo pragmatico a garantire, nella gran parte dei casi, l'adeguamento dell'Accordo all'evoluzione della giurisprudenza della Corte sarebbe ben presente e solamente in casi eccezionali si ricorrerebbe ad una "smentita" di quella giurisprudenza tramite modifica del Regolamento. Ove quella modifica non fosse concretamente operabile, o per l'ancoraggio dell'interpretazione data dalla Corte ai principi del Trattato, o per la più banale impossibilità di raggiungere le maggioranze a ciò necessarie nelle istituzioni co-legislative, la scelta sarebbe a questo punto al governo e al parlamento britannici. In ogni caso, non si tratterebbe di un'alternativa drammatica.

Da un lato, infatti, si aprirebbe l'ipotesi di confermare, nonostante tutto, l'adeguamento del sistema richiesto dalla parte UE: in fondo, in tal modo, si potrebbe ben argomentare, nei confronti dei critici dell'Unione a priori, che quell'adeguamento è sovranamente consentito dalla parte britannica tramite una deliberazione sovrana del Parlamento di Westminster, cui anche la common law è abituata a piegarsi.

Dall'altro lato, in presenza di una rete di sicurezza costituita dalla Convenzione di Lugano o da un terreno comune di fondo determinato da due regimi unilaterali grosso modo equivalenti, quel dibattito parlamentare potrà svolgersi con la reale prospettiva di un esito alternativo, non catastrofico.

24. Giunti al punto in cui siamo, proviamo a chiudere queste riflessioni in una chiave semiseria, come abbiamo aperto questo lavoro, e così com'è in fondo tutta la vicenda della Brexit: a metà tra dramma e commedia.

E se fosse tutto un equivoco en travesti?

Se l'apparente nonchalance con cui il governo britannico e il negoziatore dell'Unione hanno considerato l'obiettivo di continuare a garantire le condizioni tecnico giuridiche delle relazioni sociali ed economiche tra gli individui across the Channel fosse solo una prova... per infine riannodare una relazione che deve continuare- almeno in quest'ambito, against all odds?

Nulla in fondo, se non un'ostinata (cieca) perseveranza ideologica nel recidere ogni legame con

${ }^{154}$ Nella prospettiva radicalmente critica sul rapporto tra giurisprudenza e principi di common law sostanzialmente assunta da molti e ben esemplificata dal saggio di T.C. HARTLEY già citato in apertura di questo lavoro. 
...la perfida Unione, da un lato, ed un risentito livore del partner abbandonato, dall'altro, spiegherebbe perché rinunciare ad un meccanismo settoriale che garantisca sostanziale continuità, anche ove i termini delle complessive relazioni commerciali non dovessero definiti in maniera soddisfacente in un accordo globale entro la data ghigliottina del 31 dicembre 2020. Perché non c'è alcun dubbio che l'interesse di entrambe le parti di questa commedia è certo quello di favorire il più possibile il mantenimento della stabilità delle situazioni giuridiche e la prevedibilità delle relazioni in materia civile e commerciale, obiettivo che solo una facile circolazione dei giudicati può garantire.

Pensiamo allora che quel disinteresse di cui le parti negoziali hanno fatto mostra sinora sia solo un gioco e che alla fine, con le parole che Rosalind rivolge ad Orlando in As you like it, ci sentiremo dire: "Believe then, if you please, that I can do strange things. I have, since I was three year old, conversed with a magician, most profound in his art and yet not damnabler" ${ }^{\prime 15}$ così che infine, Hymen, la... divinità delle unioni, possa chiudere anche questa commedia annunciando "Then is there mirth in heaven, / When earthly things made even / Atone together"156.

${ }^{155}$ W. Shakespeare, As you like it, (Act V. Scene ii. 63-66).

${ }^{156}$ W. Shakespeare, As you like it, (Act V. Scene iv. 114-116). 